\title{
A refined integro-surface energy-based model for vibration of magnetically actuated double-nanowire-systems carrying electric current
}

\author{
Keivan Kiani ${ }^{\mathrm{a}, *}$ \\ ${ }^{a}$ Department of Civil Engineering, K.N. Toosi University of Technology, \\ P.O. Box 15875-4416, Valiasr Ave., Tehran, Iran
}

\begin{abstract}
A novel surface energy-based model is developed to examine more precisely vibrations of current-carrying double-nanowire-systems immersed in a longitudinal magnetic field. Using Biot-Savart and Lorentz laws, a more refined version of interwire interactional magnetic forces is presented. By employing Rayleigh beam theory, the equations of motion are derived. In fact, these are coupled integro-differential equations which are more accurate with respect to those of the previously developed models. For simply supported and clamped nanosystems, governing equations are analyzed via assumed mode method. The effects of interwire distance, slenderness ratio, electric current, magnetic field strength, and surface effect on the fundamental frequency are addressed carefully. The obtained results display the importance of exploiting the refined model for vibration analysis of nanosystems with low interwire distance, high electric current, and high magnetic field strength.

Keywords: Transverse vibrations; Double-nanowire-systems; Direct electric current; Longitudinal magnetic field; Surface energy effect; Assumed mode method.
\end{abstract}

\footnotetext{
*Corresponding author. Tel: +98 21 88779473; Fax: +98 2188779476.

Email address: k_kiani@kntu.ac.ir; keivankiani@yahoo.com (Keivan Kiani)
} 


\section{Introduction}

The assembly of nanowires (NWs) into nanodevices and nanoscale circuits brings about diverse applications in nanoelectronics $[1,2]$, nanogenerators [3-5], nanophotonics [6-8], and nano-sized sensors [9-11]. For most of these purposes, electric currents would pass through the NWs and they would interact dynamically due to the resulted magnetic fields. As a result, realizing true vibrations of group of current-carrying NWs helps us to design them optimally for the desired functionality, serviceability, and safety. A preliminary step towards this goal is to examine vibrations of double current-carrying nanowires (DCCNWs) as the basic building block of ensembles of nanowires. To generalize the problem, a longitudinal magnetic field is also considered and it is noted that magnetically actuated NWs carrying current could also have potential applications in the upcoming nanoscale magnetometers. Thereby, this paper deals on vibrations of magnetically actuated DCCNWs.

For an individual current-carrying NW (CCNW) subjected to a longitudinal magnetic field, any lateral vibration of the nanostructure leads to exertion of a magnetic force in which could endanger its stability depends on the magnitudes of the electric current, strength of the magnetic field, and geometry properties of the NW [12-14]. For magnetically actuated DCCNWs, the scenario of vibrations and instabilities would be completely different from that of the individual NWs. When an electric current passes through a double-nanowiresystem, each nanowire is actuated by the generated magnetic field of its adjacent wire. The induced magnetic fields at the locations of the deformed NWs could be evaluated using Biot-Savart's law whereas the exerted magnetic forces on them are commonly computed via Lorentz's law. In the previous explorations $[15,16]$, vibrations and dynamic instability of lengthy DCCNWs with and without application of magnetic field were studied. Since the length of the NWs was large enough, their bending rigidities were provoked and the exerted magnetic field could be easily evaluated based on the infinite length assumption. These simplifications in the suggested models would not be satisfactory when examination of vibrations of a finite length magnetically affected DCCNWs is of concern. One of the chief 
objectives of this study is to develop a more sophisticated model to take into account both bending rigidity in the equations of motion and the NWs' finite length in the formulations of the interwire dynamic magnetic forces.

As the dimensions of the solid structure decrease, the ratio of the surface area to the bulk volume increases. For nanoscale structures, the surface energy is high enough that its effect cannot be ignored in their total potential energy. The classical continuum theory cannot explain such a phenomenon since it is basically developed for mechanical behavior of macroscale structures which the surface energy can be safely excluded from their formulations. Gurtin and Murdoch [17-19] suggested an advanced elasticity theory to incorporate the surface energy into the equations of motion of the solid nanostructures. In constructing their model, it was displayed that the surface is a very thin layer with negligible thickness that adhered tightly to the underlying bulk zone. Therefore, the displacements and the resulting strain fields within the surface layer are the same as those of the bulk at its vicinity; however, the constitutive relations of the surface layer are basically different from those of the bulk as displayed by the Gurtin-Murdoch model [17-19]. Until now, the surface elasticity theory of Gurtin-Murdoch has been extensively implemented to inspect statics [20-25], vibrations [26-34], buckling and postbuckling [35-41] behaviors of nanoscale beams and nanowires.

In this article, free transverse vibrations of DCCNWs in the presence of a longitudinal magnetic field are going to be investigated numerically. Using Biot-Savart law, approximate and exact magnetic forces on the deformed nanowires carrying current are evaluated. By employing Gurtin-Murdoch elasticity theory in conjunction with the Rayleigh beam theory, the problem is formulated and analyzed via assumed mode method (AMM). For both simply supported and fully clamped ends, the capability of the approximate model in predicting the results of the exact model is displayed. Subsequently, the roles of the interwire distance, slenderness ratio, electric current, magnetic field strength, and surface energy effect on the free vibration behavior of the nanosystem are addressed. The suggested model based on 
the exact formulations would provide useful insights for more accurate dynamic analysis of vertically aligned CCNWs.

\section{Statement of the problem}

Consider a system of double nanowires with length $l_{b}$ and the interwire distance $d$ which is used to carry electric current $I_{0}$ (see Fig. 1). The nanosystem is subjected to a longitudinal magnetic field of the form: $\mathbf{B}=B_{0} \mathbf{e}_{x_{1}}$ or $\mathbf{B}=B_{0} \mathbf{e}_{x_{2}}$ where $B_{0}$ denotes the strength of the magnetic field. The Cartesian coordinate systems associated with the nanowires have been demonstrated in Fig. 1. Both electric current and magnetic field strength do not vary with time. Herein, we are interested in exploring free vibrations of such a nanosystem in the context of the surface elasticity theory of Gurtin-Murdoch. To this end, the interactional dynamic magnetic forces on the deformed nanowires should be accurately evaluated.

\section{The interwire interactional magnetic forces: Approximate vs. exact formu-}

\section{lations}

The electric current passes through the $j$ th deformed CCNW is given by [12-14]:

$$
\mathbf{I}_{j}=I_{0}\left(\mathbf{e}_{x_{j}}+\frac{\partial u_{y_{j}}}{\partial x_{j}} \mathbf{e}_{y_{j}}+\frac{\partial u_{z_{j}}}{\partial x_{j}} \mathbf{e}_{z_{j}}\right) ; j=1,2,
$$

where $u_{y_{j}}$ and $u_{z_{j}}$ denote the transverse displacements of the $j$ th CCNW along the $y$ axis and the $z$ axis, respectively. Each CCNW is actuated by the externally applied longitudinal magnetic field as well as by the magnetic field of its adjacent deformed CCNW. The total magnetic field at the location of the $j$ th CCNW is calculated using the Biot-Savart law:

$$
\mathbf{B}_{j}=B_{0} \mathbf{e}_{x_{j}}+\frac{\mu_{0}}{4 \pi} \int_{0}^{l_{b}} \frac{\mathbf{I}_{3-j} \times \mathbf{r}_{3-j}}{\left\|\mathbf{r}_{3-j}\right\|^{3}} \mathrm{~d} s_{j},
$$

where $\mu_{0}=4 \pi \times 10^{-7} \frac{\mathrm{Tm}}{\mathrm{A}}$ is the permeability of free space, and $\|$.$\| is the norm sign, and the$ position vector $\mathbf{r}_{j} ; j=1,2$ is provided by: $\mathbf{r}_{j}=(-1)^{j-1}\left[\left(x_{2}-x_{1}\right) \mathbf{e}_{x_{1}}+\left(d+\Delta u_{y}\right) \mathbf{e}_{y_{1}}+\Delta u_{z} \mathbf{e}_{z_{1}}\right]$ where $\Delta u_{y}=u_{y_{2}}-u_{y_{1}}$ and $\Delta u_{z}=u_{z_{2}}-u_{z_{1}}$. By employing the Lorentz's law, the magnetic force per unit length of the $j$ th CCNW is calculated by: $\mathbf{f}_{m_{j}}=\mathbf{I}_{j} \times \mathbf{B}_{j} ; j=1,2$. 


\subsection{An approximation formulation}

Let's assume that the length of both CCNWs are large. Using Eq. (2), the magnetic fields at the location of deformed CCNWs take the following form:

$$
\begin{aligned}
\mathbf{B}_{1}= & \left(B_{0}-\frac{\mu_{0} I_{0}}{2 \pi} \frac{\Delta w \frac{\partial v_{2}}{\partial x_{2}}-(d+\Delta v) \frac{\partial w_{2}}{\partial x_{2}}}{(d+\Delta v)^{2}+(\Delta w)^{2}}\right) \mathbf{e}_{x_{1}} \\
& +\left(\frac{\mu_{0} I_{0}}{2 \pi} \frac{\Delta w}{(d+\Delta v)^{2}+(\Delta w)^{2}}\right) \mathbf{e}_{y_{1}}-\left(\frac{\mu_{0} I_{0}}{2 \pi} \frac{d+\Delta v}{(d+\Delta v)^{2}+(\Delta w)^{2}}\right) \mathbf{e}_{z_{1}}, \\
\mathbf{B}_{2}= & \left(B_{0}+\frac{\mu_{0} I_{0}}{2 \pi} \frac{\Delta w \frac{\partial v_{1}}{\partial x_{1}}-(d+\Delta v) \frac{\partial w_{1}}{\partial x_{1}}}{(d+\Delta v)^{2}+(\Delta w)^{2}}\right) \mathbf{e}_{x_{2}} \\
& -\left(\frac{\mu_{0} I_{0}}{2 \pi} \frac{\Delta w}{(d+\Delta v)^{2}+(\Delta w)^{2}}\right) \mathbf{e}_{y_{2}}+\left(\frac{\mu_{0} I_{0}}{2 \pi} \frac{d+\Delta v}{(d+\Delta v)^{2}+(\Delta w)^{2}}\right) \mathbf{e}_{z_{2}} .
\end{aligned}
$$

By considering: $\frac{d+\Delta v}{(d+\Delta v)^{2}+(\Delta w)^{2}} \approx \frac{1}{d}\left(1-\frac{\Delta v}{d}+\left(\frac{\Delta v}{d}\right)^{2}\right)$ and $\frac{\Delta w}{(d+\Delta v)^{2}+(\Delta w)^{2}} \approx$ $\frac{\Delta w}{d^{2}}\left(1-\left(\frac{\Delta w}{d}\right)^{2}\right)$, and then substituting these relations into Eqs. (3a) and (3b) through implementing the Lorentz's formulas, the exerted magnetic force on the $j$ th laterally deformed CCNWs is evaluated as:

$$
\mathbf{f}_{m_{j}}=f_{m_{x j}} \mathbf{e}_{x_{j}}+f_{m_{y j}} \mathbf{e}_{y_{j}}+f_{m_{z j}} \mathbf{e}_{z_{j}} ; j=1,2,
$$

where

$$
\begin{aligned}
& f_{m_{x 1}} \approx \frac{\mu_{0} I_{0}^{2}}{2 \pi d}\left[\frac{\partial v_{1}}{\partial x_{1}}\left(1+\frac{\Delta v}{d}+\left(\frac{\Delta v}{d}\right)^{2}\right)-\frac{\partial w_{2}}{\partial x_{2}}\left(\frac{\Delta w}{d}\right)\left(1-\left(\frac{\Delta w}{d}\right)^{2}\right)\right], \\
& f_{m_{y 1}} \approx B_{0} I_{0} \frac{\partial w_{1}}{\partial x_{1}}-\frac{\mu_{0} I_{0}^{2}}{2 \pi d}\left[\begin{array}{c}
\frac{\partial v_{1}}{\partial x_{1}} \frac{\partial w_{1}}{\partial x_{1}}\left(\frac{\Delta w}{d}\right)\left(1-\left(\frac{\Delta w}{d}\right)^{2}\right)+ \\
\left(1+\left(\frac{\partial w_{1}}{\partial x_{1}}\right)^{2}\right)\left(1+\frac{\Delta v}{d}+\left(\frac{\Delta v}{d}\right)^{2}\right)
\end{array}\right] \\
& f_{m_{z 1}} \approx-B_{0} I_{0} \frac{\partial v_{1}}{\partial x_{1}}+\frac{\mu_{0} I_{0}^{2}}{2 \pi d}\left[\begin{array}{l}
\frac{\partial v_{1}}{\partial x_{1}} \frac{\partial w_{1}}{\partial x_{1}}\left(1+\frac{\Delta v}{d}+\left(\frac{\Delta v}{d}\right)^{2}\right)+ \\
\left(1+\left(\frac{\partial v_{1}}{\partial x_{1}}\right)^{2}\right)\left(\frac{\Delta w}{d}\right)\left(1-\left(\frac{\Delta w}{d}\right)^{2}\right)
\end{array}\right],
\end{aligned}
$$


and

$$
\begin{aligned}
& f_{m_{x 2}} \approx \frac{\mu_{0} I_{0}^{2}}{2 \pi d}\left[\frac{\partial v_{2}}{\partial x_{2}}\left(1-\frac{\Delta v}{d}+\left(\frac{\Delta v}{d}\right)^{2}\right)+\frac{\partial w_{2}}{\partial x_{2}}\left(\frac{\Delta w}{d}\right)\left(1-\left(\frac{\Delta w}{d}\right)^{2}\right)\right], \\
& f_{m_{y 2}} \approx B_{0} I_{0} \frac{\partial w_{2}}{\partial x_{2}}+\frac{\mu_{0} I_{0}^{2}}{2 \pi d}\left[\begin{array}{c}
\frac{\partial v_{2}}{\partial x_{2}} \frac{\partial w_{2}}{\partial x_{2}}\left(\frac{\Delta w}{d}\right)\left(1-\left(\frac{\Delta w}{d}\right)^{2}\right)- \\
\left(1+\left(\frac{\partial w_{2}}{\partial x_{2}}\right)^{2}\right)\left(1-\frac{\Delta v}{d}+\left(\frac{\Delta v}{d}\right)^{2}\right)
\end{array}\right] \\
& f_{m_{z 2}} \approx-B_{0} I_{0} \frac{\partial v_{2}}{\partial x_{2}}+\frac{\mu_{0} I_{0}^{2}}{2 \pi d}\left[\begin{array}{l}
\frac{\partial v_{2}}{\partial x_{2}} \frac{\partial w_{2}}{\partial x_{2}}\left(1-\frac{\Delta v}{d}+\left(\frac{\Delta v}{d}\right)^{2}\right)- \\
\left(1+\left(\frac{\partial v_{2}}{\partial x_{2}}\right)^{2}\right)\left(\frac{\Delta w}{d}\right)\left(1-\left(\frac{\Delta w}{d}\right)^{2}\right)
\end{array}\right]
\end{aligned}
$$

Through neglecting the products of partial derivatives of deflections as well as the square of ratio of relative deflections to the interwire distance, the magnetic forces applied on the deformed CCNWs are simplified to:

$$
\begin{aligned}
\mathbf{f}_{m_{1}}= & \frac{\mu_{0} I_{0}^{2}}{2 \pi d} \frac{\partial v_{1}}{\partial x_{1}} \mathbf{e}_{x_{1}}+\left(B_{0} I_{0} \frac{\partial w_{1}}{\partial x_{1}}+\frac{\mu_{0} I_{0}^{2}}{2 \pi d}\left(1-\frac{\triangle v}{d}\right)\right) \mathbf{e}_{y_{1}} \\
& +\left(-B_{0} I_{0} \frac{\partial v_{1}}{\partial x_{1}}+\frac{\mu_{0} I_{0}^{2}}{2 \pi d}\left(\frac{\triangle w}{d}\right)\right) \mathbf{e}_{z_{1}}, \\
\mathbf{f}_{m_{2}}= & \frac{\mu_{0} I_{0}^{2}}{2 \pi d} \frac{\partial v_{2}}{\partial x_{2}} \mathbf{e}_{x_{2}}+\left(B_{0} I_{0} \frac{\partial w_{2}}{\partial x_{2}}-\frac{\mu_{0} I_{0}^{2}}{2 \pi d}\left(1-\frac{\triangle v}{d}\right)\right) \mathbf{e}_{y_{2}} \\
& +\left(-B_{0} I_{0} \frac{\partial v_{2}}{\partial x_{2}}-\frac{\mu_{0} I_{0}^{2}}{2 \pi d}\left(\frac{\triangle w}{d}\right)\right) \mathbf{e}_{z_{2}} .
\end{aligned}
$$

By ignoring the static terms of magnetic forces in Eqs. (7a) and (7b), the dynamic magnetic forces that exert laterally on the CCNWs of the nanosystem are evaluated by:

$$
\begin{gathered}
\mathbf{f}_{m_{1}}=\left(B_{0} I_{0} \frac{\partial w_{1}}{\partial x_{1}}-\frac{\mu_{0} I_{0}^{2}}{2 \pi d^{2}}\left(v_{2}-v_{1}\right)\right) \mathbf{e}_{y_{1}}+\left(-B_{0} I_{0} \frac{\partial v_{1}}{\partial x_{1}}+\frac{\mu_{0} I_{0}^{2}}{2 \pi d^{2}}\left(w_{2}-w_{1}\right)\right) \mathbf{e}_{z_{1}}, \\
\mathbf{f}_{m_{2}}=\left(B_{0} I_{0} \frac{\partial w_{2}}{\partial x_{2}}+\frac{\mu_{0} I_{0}^{2}}{2 \pi d^{2}}\left(v_{2}-v_{1}\right)\right) \mathbf{e}_{y_{2}}-\left(B_{0} I_{0} \frac{\partial v_{2}}{\partial x_{2}}+\frac{\mu_{0} I_{0}^{2}}{2 \pi d^{2}}\left(w_{2}-w_{1}\right)\right) \mathbf{e}_{z_{2}}
\end{gathered}
$$




\subsection{An exact formulation}

By introducing Eq. (1) to Eqs. (2a) and (2b), the magnetic fields at the neighboring of the CCNWs are exactly calculated as:

$$
\begin{aligned}
\mathbf{B}_{1}= & \left(B_{0}-\frac{\mu_{0} I_{0}}{4 \pi} \int_{0}^{l_{b}} \frac{\Delta w \frac{\partial v_{2}}{\partial x_{2}}-(d+\Delta v) \frac{\partial w_{2}}{\partial x_{2}}}{\left(\left(x_{2}-x_{1}\right)^{2}+(d+\Delta v)^{2}+(\Delta w)^{2}\right)^{\frac{3}{2}}} \mathrm{~d} x_{2}\right) \mathbf{e}_{x_{1}}- \\
& \left(\frac{\mu_{0} I_{0}}{4 \pi} \int_{0}^{l_{b}} \frac{\left(x_{2}-x_{1}\right) \frac{\partial w_{2}}{\partial x_{2}}-\Delta w}{\left(\left(x_{2}-x_{1}\right)^{2}+(d-\Delta v)^{2}+(\Delta w)^{2}\right)^{\frac{3}{2}}} \mathrm{~d} x_{2}\right) \mathbf{e}_{y_{1}}- \\
& \left(\frac{\mu_{0} I_{0}}{4 \pi} \int_{0}^{l_{b}} \frac{(d+\Delta v)-\left(x_{2}-x_{1}\right) \frac{\partial v_{2}}{\partial x_{2}}}{\left(\left(x_{2}-x_{1}\right)^{2}+(d+\Delta v)^{2}+(\Delta w)^{2}\right)^{\frac{3}{2}}} \mathrm{~d} x_{1}\right) \mathbf{e}_{z_{1}},
\end{aligned}
$$

and

$$
\begin{aligned}
\mathbf{B}_{2}= & \left(B_{0}+\frac{\mu_{0} I_{0}}{4 \pi} \int_{0}^{l_{b}} \frac{\Delta w \frac{\partial v_{1}}{\partial x_{1}}-(d+\Delta v) \frac{\partial w_{1}}{\partial x_{1}}}{\left(\left(x_{2}-x_{1}\right)^{2}+(d+\Delta v)^{2}+(\Delta w)^{2}\right)^{\frac{3}{2}}} \mathrm{~d} x_{1}\right) \mathbf{e}_{x_{2}}+ \\
& \left(\frac{\mu_{0} I_{0}}{4 \pi} \int_{0}^{l_{b}} \frac{\left(x_{2}-x_{1}\right) \frac{\partial w_{1}}{\partial x_{1}}-\Delta w}{\left(\left(x_{2}-x_{1}\right)^{2}+(d+\Delta v)^{2}+(\Delta w)^{2}\right)^{\frac{3}{2}}} \mathrm{~d} x_{1}\right) \mathbf{e}_{y_{2}}+ \\
& \left(\frac{\mu_{0} I_{0}}{4 \pi} \int_{0}^{l_{b}} \frac{(d+\Delta v)-\left(x_{2}-x_{1}\right) \frac{\partial v_{1}}{\partial x_{1}}}{\left(\left(x_{2}-x_{1}\right)^{2}+(d+\Delta v)^{2}+(\Delta w)^{2}\right)^{\frac{3}{2}}} \mathrm{~d} x_{1}\right) \mathbf{e}_{z_{2}} .
\end{aligned}
$$

Using Lorentz's formula in view of Eqs. (9) and (10), the components of the magnetic forces on the CCNWs immersed in a longitudinal magnetic field are obtained:

$$
\begin{gathered}
f_{m_{x 1}}=-\frac{\mu_{0} I_{0}^{2}}{4 \pi} \int_{0}^{l_{b}} \frac{(d+\Delta v) \frac{\partial v_{1}}{\partial x_{1}}+\left(x_{2}-x_{1}\right)\left(\frac{\partial v_{1}}{\partial x_{1}} \frac{\partial v_{2}}{\partial x_{2}}+\frac{\partial w_{1}}{\partial x_{1}} \frac{\partial w_{2}}{\partial x_{2}}\right)+\Delta w \frac{\partial w_{1}}{\partial x_{1}}}{\left(\left(x_{2}-x_{1}\right)^{2}+(d+\Delta v)^{2}+(\Delta w)^{2}\right)^{\frac{3}{2}}} \mathrm{~d} x_{2}, \\
f_{m_{y 1}}=B_{0} I_{0} \frac{\partial w_{1}}{\partial x_{1}}-\frac{\mu_{0} I_{0}^{2}}{4 \pi} \int_{0}^{l_{b}} \frac{\Delta w \frac{\partial w_{1}}{\partial x_{1}} \frac{\partial v_{2}}{\partial x_{2}}+(d+\Delta v)\left(1+\frac{\partial w_{1}}{\partial x_{1}} \frac{\partial w_{2}}{\partial x_{2}}\right)+\left(x_{2}-x_{1}\right) \frac{\partial v_{2}}{\partial x_{2}}}{\left(\left(x_{2}-x_{1}\right)^{2}+(d+\Delta v)^{2}+(\Delta w)^{2}\right)^{\frac{3}{2}}} \mathrm{~d} x_{2},(11 \mathrm{~b}) \\
f_{m_{z 1}}=-B_{0} I_{0} \frac{\partial v_{1}}{\partial x_{1}}-\frac{\mu_{0} I_{0}^{2}}{4 \pi} \int_{0}^{l_{b}} \frac{\left(x_{2}-x_{1}\right) \frac{\partial w_{2}}{\partial x_{2}}+\Delta w\left(1+\frac{\partial v_{1}}{\partial x_{1}} \frac{\partial v_{2}}{\partial x_{2}}\right)+(d+\Delta v) \frac{\partial w_{1}}{\partial x_{1}} \frac{\partial v_{2}}{\partial x_{2}}}{\left(\left(x_{2}-x_{1}\right)^{2}+(d+\Delta v)^{2}+(\Delta w)^{2}\right)^{\frac{3}{2}}} \mathrm{~d} x_{2},(11 \mathrm{c})
\end{gathered}
$$




$$
\begin{gathered}
f_{m_{x 2}}=\frac{\mu_{0} I_{0}^{2}}{4 \pi} \int_{0}^{l_{b}} \frac{(d+\Delta v) \frac{\partial v_{2}}{\partial x_{2}}-\left(x_{2}-x_{1}\right)\left(\frac{\partial v_{1}}{\partial x_{1}} \frac{\partial v_{2}}{\partial x_{2}}+\frac{\partial w_{1}}{\partial x_{1}} \frac{\partial w_{2}}{\partial x_{2}}\right)-\Delta w \frac{\partial w_{2}}{\partial x_{2}}}{\left(\left(x_{2}-x_{1}\right)^{2}+(d+\Delta v)^{2}+(\Delta w)^{2}\right)^{\frac{3}{2}}} \mathrm{~d} x_{1} \\
f_{m_{y 2}}=B_{0} I_{0} \frac{\partial w_{2}}{\partial x_{2}}+\frac{\mu_{0} I_{0}^{2}}{4 \pi} \int_{0}^{l_{b}} \frac{\Delta w \frac{\partial v_{1}}{\partial x_{1}} \frac{\partial w_{2}}{\partial x_{2}}-(d+\Delta v)\left(1+\frac{\partial w_{1}}{\partial x_{1}} \frac{\partial w_{2}}{\partial x_{2}}\right)+\left(x_{2}-x_{1}\right) \frac{\partial v_{1}}{\partial x_{1}}}{\left(\left(x_{2}-x_{1}\right)^{2}+(d+\Delta v)^{2}+(\Delta w)^{2}\right)^{\frac{3}{2}}} \mathrm{~d} x_{1},(1 \\
f_{m_{z 2}}=-B_{0} I_{0} \frac{\partial v_{2}}{\partial x_{2}}+\frac{\mu_{0} I_{0}^{2}}{4 \pi} \int_{0}^{l_{b}} \frac{\left(x_{2}-x_{1}\right) \frac{\partial w_{1}}{\partial x_{1}}-\Delta w\left(1+\frac{\partial v_{1}}{\partial x_{1}} \frac{\partial v_{2}}{\partial x_{2}}\right)+(d+\Delta v) \frac{\partial w_{1}}{\partial x_{1}} \frac{\partial v_{2}}{\partial x_{2}}}{\left(\left(x_{2}-x_{1}\right)^{2}+(d+\Delta v)^{2}+(\Delta w)^{2}\right)^{\frac{3}{2}}} \mathrm{~d} x_{1} \cdot(11)
\end{gathered}
$$

One can rationally assume that: $\Delta v \ll d$ and $\Delta w \ll d$. By ignoring the products of the first derivatives of displacements, and excluding the static terms, the dynamical magnetic forces on the deformed CCNWs are evaluated as follows:

$$
\begin{aligned}
\mathbf{f}_{m_{1}}= & \left(\frac{\mu_{0} I_{0}^{2}}{4 \pi} \int_{0}^{l_{b}} \frac{d}{\left(\left(x_{2}-x_{1}\right)^{2}+d^{2}\right)^{\frac{3}{2}}} \frac{\partial v_{1}}{\partial x_{1}} \mathrm{~d} x_{2}\right) \mathbf{e}_{x_{1}}+ \\
& \left(B_{0} I_{0} \frac{\partial w_{1}}{\partial x_{1}}-\frac{\mu_{0} I_{0}^{2}}{4 \pi} \int_{0}^{l_{b}} \frac{\left(x_{2}-x_{1}\right) \frac{\partial v_{2}}{\partial x_{2}}-\Delta v}{\left(\left(x_{2}-x_{1}\right)^{2}+d^{2}\right)^{\frac{3}{2}}} \mathrm{~d} x_{2}\right) \mathbf{e}_{y_{1}}+ \\
& \left(-B_{0} I_{0} \frac{\partial v_{1}}{\partial x_{1}}-\frac{\mu_{0} I_{0}^{2}}{4 \pi} \int_{0}^{l_{b}} \frac{\left(x_{2}-x_{1}\right) \frac{\partial w_{2}}{\partial x_{2}}-\Delta w}{\left(\left(x_{2}-x_{1}\right)^{2}+d^{2}\right)^{\frac{3}{2}}} \mathrm{~d} x_{2}\right) \mathbf{e}_{z_{1}}, \\
\mathbf{f}_{m_{2}=} & \left(\frac{\mu_{0} I_{0}^{2}}{4 \pi} \int_{0}^{l_{b}} \frac{\partial v_{2}}{\left(\left(x_{2}-x_{1}\right)^{2}+d^{2}\right)^{\frac{3}{2}}} \mathrm{~d} x_{1}\right) \mathbf{e}_{x_{2}}+ \\
& \left(B_{0} I_{0} \frac{\partial w_{2}}{\partial x_{2}}+\frac{\mu_{0} I_{0}^{2}}{4 \pi} \int_{0}^{l_{b}} \frac{\left(x_{2}-x_{1}\right) \frac{\partial v_{1}}{\partial x_{1}}-\Delta v}{\left(\left(x_{2}-x_{1}\right)^{2}+d^{2}\right)^{\frac{3}{2}}} \mathrm{~d} x_{1}\right) \mathbf{e}_{y_{2}}+ \\
& \left(-B_{0} I_{0} \frac{\partial v_{2}}{\partial x_{2}}+\frac{\mu_{0} I_{0}^{2}}{4 \pi} \int_{0}^{l_{b}} \frac{\left(x_{2}-x_{1}\right) \frac{\partial w_{1}}{\partial x_{1}}-\Delta w}{\left(\left(x_{2}-x_{1}\right)^{2}+d^{2}\right)^{\frac{3}{2}}} \mathrm{~d} x_{1}\right) \mathbf{e}_{z_{2}} .
\end{aligned}
$$




\section{Establishment of a novel integro-surface energy-based model}

\subsection{The constitutive relations of the surface layer and the bulk}

Using theory of elasticity of Gurtin-Murdoch [17-19], the constitutive relations of the surface layer are given by:

$$
\begin{gathered}
\tau_{\alpha \beta}=\tau_{0} \delta_{\alpha \beta}+\tau_{0} \frac{\partial u_{\alpha}}{\partial \beta}+2\left(\mu_{0}-\tau_{0}\right) \epsilon_{\alpha \beta}+\left(\lambda_{0}+\tau_{0}\right) \epsilon_{\gamma \gamma} \delta_{\alpha \beta} ; \alpha, \beta, \gamma=x, y, z, \\
\tau_{x \alpha}=\tau_{0} \frac{\partial u_{\alpha}}{\partial x} ; \alpha=y, z
\end{gathered}
$$

where $\tau_{\alpha \beta}$ is the surface stress tensor, $\epsilon_{\alpha \beta}$ is the surface strain tensor, $u_{\alpha}$ is the displacement along the $\alpha$ direction, $\lambda_{0}$ and $\mu_{0}$ are the Lame's constants of the surface layer, $\tau_{0}$ is the residual surface stress under unconstrained conditions, and $\delta_{\alpha \beta}$ denotes the Kronecker delta. By employing the Rayleigh beam theory, the displacements of the $m$ th nanowire are provided by: $u_{x_{m}}=-\left(z_{m} \frac{\partial w_{m}\left(x_{m}, t\right)}{\partial x_{m}}+y_{m} \frac{\partial v_{m}\left(x_{m}, t\right)}{\partial x_{m}}\right), u_{y_{m}}=v_{m}\left(x_{m}, t\right), u_{z_{m}}=w_{m}\left(x_{m}, t\right)$. By introducing these displacements to Eq. (13), the only existing stresses of the $m$ th NW's surface layer would be:

$$
\tau_{x x_{m}}=\tau_{0}-\left(\lambda_{0}+2 \mu_{0}\right)\left(z_{m} \frac{\partial^{2} w_{m}}{\partial x_{m}^{2}}+y_{m} \frac{\partial^{2} v_{m}}{\partial x_{m}^{2}}\right), \tau_{x y_{m}}=n_{y_{m}} \tau_{0} \frac{\partial v_{m}}{\partial x_{m}}, \quad \tau_{x z_{m}}=n_{z_{m}} \tau_{0} \frac{\partial w_{m}}{\partial x_{m}}
$$

where $\tau_{x x_{m}}$ is the longitudinal normal stress of the surface layer, $\tau_{x y_{m}}$ and $\tau_{x z_{m}}$ are the shear stresses, and $n_{y_{m}}$ and $n_{y_{m}}$ are the components of the unit outward normal vector of the surface layer of the $m$ th NW.

To construct more accurate constitutive relations for the bulk, it is assumed that the stress components $\sigma_{y y_{m}}$ and $\sigma_{z z_{m}}$ within the bulk would vary linearly between their corresponding surface values. By exploiting the generalized Hook's law, the longitudinal stress of the bulk of the $m$ th NW would be expressed by:

$$
\begin{aligned}
\sigma_{x x_{m}}= & -z_{m}\left(\left(E_{b}-\frac{2 \tau_{0} \nu_{b}}{D_{0}}\right) \frac{\partial^{2} w_{m}}{\partial x_{m}^{2}}+\frac{2 \rho_{0} \nu_{b}}{D_{0}} \frac{\partial^{2} w_{m}}{\partial t^{2}}\right) \\
& -y_{m}\left(\left(E_{b}-\frac{2 \tau_{0} \nu_{b}}{D_{0}}\right) \frac{\partial^{2} v_{m}}{\partial x_{m}^{2}}+\frac{2 \rho_{0} \nu_{b}}{D_{0}} \frac{\partial^{2} v_{m}}{\partial t^{2}}\right),
\end{aligned}
$$


where $\nu_{b}$ is the Poisson's ratio of the bulk, $E_{b}$ is the elastic modulus of the bulk, $\rho_{0}$ is the mass per unit area of the surface, and $D_{0}$ represents the diameter of the CCNWs,

\subsection{Development of equations of motion of magnetically actuated DCCNWs}

By taking an infinitesimal element of a magnetically actuated CCNW, depicting the free body diagram of such an element, and applying the Newton's second law, the transverse equations of motion of the nanosystem as a function of the bending moments and the shear forces within the CCNWs are obtained as follows:

$$
\begin{aligned}
& \frac{\partial M_{b_{z 1}}}{\partial x_{1}}+\int_{\mathcal{S}} y_{1} \frac{\partial \tau_{x x_{1}}}{\partial x_{1}} \mathrm{~d} \mathcal{S}-Q_{b_{y 1}}=\int_{A_{b}} \rho_{b} \frac{\partial^{2} u_{x_{1}}}{\partial t^{2}} y_{1} \mathrm{~d} A+\int_{\mathcal{S}} \rho_{0} \frac{\partial^{2} u_{x_{1}}}{\partial t^{2}} y_{1} \mathrm{~d} \mathcal{S}, \\
& -\frac{\partial Q_{b_{y 1}}}{\partial x}-\int_{\mathcal{S}} \frac{\partial \tau_{x y_{1}}}{\partial x} n_{y_{1}} \mathrm{~d} \mathcal{S}-T \frac{\partial^{2} v_{1}}{\partial x_{1}^{2}}=f_{m_{y 1}}-\int_{A_{b}} \rho_{b} \frac{\partial^{2} u_{y_{1}}}{\partial t^{2}} \mathrm{~d} A-\int_{\mathcal{S}} \rho_{0} \frac{\partial^{2} u_{y_{1}}}{\partial t^{2}} n_{y_{1}} \mathrm{~d} \mathcal{S}, \\
& \frac{\partial M_{b_{y 1}}}{\partial x_{1}}+\int_{\mathcal{S}} z_{1} \frac{\partial \tau_{x x_{1}}}{\partial x_{1}} \mathrm{~d} \mathcal{S}-Q_{b_{z 1}}=\int_{A_{b}} \rho_{b} \frac{\partial^{2} u_{x_{1}}}{\partial t^{2}} z_{1} \mathrm{~d} A+\int_{\mathcal{S}} \rho_{0} \frac{\partial^{2} u_{x_{1}}}{\partial t^{2}} z_{1} \mathrm{~d} \mathcal{S}, \\
& -\frac{\partial Q_{b_{z 1}}}{\partial x}-\int_{\mathcal{S}} \frac{\partial \tau_{x z_{1}}}{\partial x} n_{z_{1}} \mathrm{~d} \mathcal{S}-T \frac{\partial^{2} w_{1}}{\partial x_{1}^{2}}=f_{m_{z 1}}-\int_{A_{b}} \rho_{b} \frac{\partial^{2} u_{z_{1}}}{\partial t^{2}} \mathrm{~d} A-\int_{\mathcal{S}} \rho_{0} \frac{\partial^{2} u_{z_{1}}}{\partial t^{2}} n_{z_{1}} \mathrm{~d} \mathcal{S}, \\
& \frac{\partial M_{b_{z 2}}}{\partial x_{2}}+\int_{\mathcal{S}} y_{2} \frac{\partial \tau_{x x_{2}}}{\partial x_{2}} \mathrm{~d} \mathcal{S}-Q_{b_{y 2}}=\int_{A_{b}} \rho_{b} \frac{\partial^{2} u_{x_{2}}}{\partial t^{2}} y_{2} \mathrm{~d} A+\int_{\mathcal{S}} \rho_{0} \frac{\partial^{2} u_{x_{2}}}{\partial t^{2}} y_{2} \mathrm{~d} \mathcal{S}, \\
& -\frac{\partial Q_{b_{y 2}}}{\partial x}-\int_{\mathcal{S}} \frac{\partial \tau_{x y_{2}}}{\partial x} n_{y_{2}} \mathrm{~d} \mathcal{S}-T \frac{\partial^{2} v_{2}}{\partial x_{2}^{2}}=f_{m_{y 2}}-\int_{A_{b}} \rho_{b} \frac{\partial^{2} u_{y_{2}}}{\partial t^{2}} \mathrm{~d} A-\int_{\mathcal{S}} \rho_{0} \frac{\partial^{2} u_{y_{2}}}{\partial t^{2}} n_{y_{2}} \mathrm{~d} \mathcal{S}, \\
& \frac{\partial M_{b_{y 2}}}{\partial x_{2}}+\int_{\mathcal{S}} z_{2} \frac{\partial \tau_{x x_{2}}}{\partial x_{2}} \mathrm{~d} \mathcal{S}-Q_{b_{z 2}}=\int_{A_{b}} \rho_{b} \frac{\partial^{2} u_{x_{2}}}{\partial t^{2}} z_{2} \mathrm{~d} A+\int_{\mathcal{S}} \rho_{0} \frac{\partial^{2} u_{x_{2}}}{\partial t^{2}} z_{2} \mathrm{~d} \mathcal{S}, \\
& -\frac{\partial Q_{b_{z 2}}}{\partial x}-\int_{\mathcal{S}} \frac{\partial \tau_{x z_{2}}}{\partial x} n_{z_{2}} \mathrm{~d} \mathcal{S}-T \frac{\partial^{2} w_{2}}{\partial x_{2}^{2}}=f_{m_{z 2}}-\int_{A_{b}} \rho_{b} \frac{\partial^{2} u_{z_{2}}}{\partial t^{2}} \mathrm{~d} A-\int_{\mathcal{S}} \rho_{0} \frac{\partial^{2} u_{z_{2}}}{\partial t^{2}} n_{z_{2}} \mathrm{~d} \mathcal{S},
\end{aligned}
$$


where $\mathrm{d} \mathcal{S}$ is the length of an infinitesimal segment of the perimeter of the cross-section of CCNWs, $T$ is the initial tensile force within the CCNWs, $M_{b_{y_{m}}} / M_{b_{z_{m}}}$ and $Q_{b_{y_{m}}} / Q_{b_{z_{m}}}$ in order represent the bending moments and the shear forces of the bulk of $m$ th CCNW pertinent to the $y_{m}$ and $z_{m}$ axes which are stated by:

$$
\begin{gathered}
M_{b_{y m}}=\int_{A} z_{m} \sigma_{x x_{m}} \mathrm{~d} A=-\left(E_{b} I_{b}-\frac{2 \tau_{0} \nu_{b} I_{b}}{D_{0}}\right) \frac{\partial^{2} w_{m}}{\partial x_{m}^{2}}-\frac{2 \nu_{b} I_{b} \tau_{0}}{D_{0}} \frac{\partial^{2} w_{m}}{\partial t^{2}}, \\
M_{b_{z m}}=\int_{A} y_{m} \sigma_{x x_{m}} \mathrm{~d} A=-\left(E_{b} I_{b}-\frac{2 \tau_{0} \nu_{b} I_{b}}{D_{0}}\right) \frac{\partial^{2} v_{m}}{\partial x_{m}^{2}}-\frac{2 \nu_{b} I_{b} \tau_{0}}{D_{0}} \frac{\partial^{2} v_{m}}{\partial t^{2}},
\end{gathered}
$$

By combining Eqs. (16a), (16c), (16e), and (16g) with Eqs. (16b), (16d), (16f), and (16h), respectively, and introducing Eqs. (17a) and (17b) to the resulting relations:

$$
\begin{aligned}
& \left(\rho_{b} A_{b}+\rho_{0} S_{0}^{*}\right) \frac{\partial^{2} v_{1}}{\partial t^{2}}-\left(\rho_{b} I_{b}+\rho_{0} I_{0}^{*}-\frac{2 \nu_{b} I_{b} \rho_{0}}{D_{0}}\right) \frac{\partial^{4} v_{1}}{\partial t^{2} \partial x_{1}^{2}}+ \\
& {\left[E_{b} I_{b}+\left(\lambda_{0}+2 \mu_{0}\right) I_{0}^{*}-\frac{2 \nu_{b} I_{b} \tau_{0}}{D_{0}}\right] \frac{\partial^{4} v_{1}}{\partial x_{1}^{4}}-\left(T+\tau_{0} S^{*}\right) \frac{\partial^{2} v_{1}}{\partial x_{1}^{2}}=f_{m_{y 1}},} \\
& \left(\rho_{b} A_{b}+\rho_{0} S_{0}^{*}\right) \frac{\partial^{2} w_{1}}{\partial t^{2}}-\left(\rho_{b} I_{b}+\rho_{0} I_{0}^{*}-\frac{2 \nu_{b} I_{b} \rho_{0}}{D_{0}}\right) \frac{\partial^{4} w_{1}}{\partial t^{2} \partial x_{1}^{2}}+ \\
& {\left[E_{b} I_{b}+\left(\lambda_{0}+2 \mu_{0}\right) I_{0}^{*}-\frac{2 \nu_{b} I_{b} \tau_{0}}{D_{0}}\right] \frac{\partial^{4} w_{1}}{\partial x_{1}^{4}}-\left(T+\tau_{0} S^{*}\right) \frac{\partial^{2} w_{1}}{\partial x_{2}^{2}}=f_{m_{z 1}},} \\
& \left(\rho_{b} A_{b}+\rho_{0} S_{0}^{*}\right) \frac{\partial^{2} v_{2}}{\partial t^{2}}-\left(\rho_{b} I_{b}+\rho_{0} I_{0}^{*}-\frac{2 \nu_{b} I_{b} \rho_{0}}{D_{0}}\right) \frac{\partial^{4} v_{2}}{\partial t^{2} \partial x_{2}^{2}+} \\
& {\left[E_{b} I_{b}+\left(\lambda_{0}+2 \mu_{0}\right) I_{0}^{*}-\frac{2 \nu_{b} I_{b} \tau_{0}}{D_{0}}\right] \frac{\partial^{4} v_{2}}{\partial x_{2}^{4}}-\left(T+\tau_{0} S^{*}\right) \frac{\partial^{2} v_{2}}{\partial x_{2}^{2}}=f_{m_{y 2}},} \\
& \left(\rho_{b} A_{b}+\rho_{0} S_{0}^{*}\right) \frac{\partial^{2} w_{2}}{\partial t^{2}}-\left(\rho_{b} I_{b}+\rho_{0} I_{0}^{*}-\frac{2 \nu_{b} I_{b} \rho_{0}}{D_{0}}\right) \frac{\partial^{4} w_{2}}{\partial t^{2} \partial x_{2}^{2}}+ \\
& {\left[E_{b} I_{b}+\left(\lambda_{0}+2 \mu_{0}\right) I_{0}^{*}-\frac{2 \nu_{b} I_{b} \tau_{0}}{D_{0}}\right] \frac{\partial^{4} w_{2}}{\partial x_{2}^{4}}-\left(T+\tau_{0} S^{*}\right) \frac{\partial^{2} w_{2}}{\partial x_{2}^{2}}=f_{m_{z 2}},}
\end{aligned}
$$

where $I_{0}^{*}=\int_{\mathcal{S}} z^{2} \mathrm{~d} \mathcal{S}$ and $S^{*}=\int_{\mathcal{S}} n_{z}^{2} \mathrm{~d} \mathcal{S}$. Eqs. (18a)-(18d) furnish us regarding transverse equations of motion of magnetically actuated DCCNWs in terms of interactional interwire magnetic forces. Based on the newly developed approximate and exact magnetic forces in section 3, the approximate and exact versions of the governing equations are established in the following parts. 


\subsubsection{An approximate model}

By substituting Eqs. (8a) and (8b) into Eqs. (18a)-(18d), the approximate form of the governing equations as a function of transverse displacements of the CCNWs is extracted as follows:

$$
\begin{aligned}
& \left(\rho_{b} A_{b}+\rho_{0} S_{0}^{*}\right) \frac{\partial^{2} v_{1}}{\partial t^{2}}-\left(\rho_{b} I_{b}+\rho_{0} I_{0}^{*}-\frac{2 \nu_{b} I_{b} \rho_{0}}{D_{0}}\right) \frac{\partial^{4} v_{1}}{\partial t^{2} \partial x_{1}^{2}}+ \\
& {\left[E_{b} I_{b}+\left(\lambda_{0}+2 \mu_{0}\right) I_{0}^{*}-\frac{2 \nu_{b} I_{b} \tau_{0}}{D_{0}}\right] \frac{\partial^{4} v_{1}}{\partial x_{1}^{4}}-\left(T+\tau_{0} S^{*}\right) \frac{\partial^{2} v_{1}}{\partial x_{1}^{2}}} \\
& -B_{0} I_{0} \frac{\partial w_{1}}{\partial x_{1}}+\frac{\mu_{0} I_{0}^{2}}{2 \pi d^{2}}\left(v_{2}-v_{1}\right)=0, \\
& \left(\rho_{b} A_{b}+\rho_{0} S_{0}^{*}\right) \frac{\partial^{2} w_{1}}{\partial t^{2}}-\left(\rho_{b} I_{b}+\rho_{0} I_{0}^{*}-\frac{2 \nu_{b} I_{b} \rho_{0}}{D_{0}}\right) \frac{\partial^{4} w_{1}}{\partial t^{2} \partial x_{1}^{2}}+ \\
& {\left[E_{b} I_{b}+\left(\lambda_{0}+2 \mu_{0}\right) I_{0}^{*}-\frac{2 \nu_{b} I_{b} \tau_{0}}{D_{0}}\right] \frac{\partial^{4} w_{1}}{\partial x_{1}^{4}}-\left(T+\tau_{0} S^{*}\right) \frac{\partial^{2} w_{1}}{\partial x_{1}^{2}}+} \\
& B_{0} I_{0} \frac{\partial v_{1}}{\partial x_{1}}-\frac{\mu_{0} I_{0}^{2}}{2 \pi d^{2}}\left(w_{2}-w_{1}\right)=0, \\
& \left(\rho_{b} A_{b}+\rho_{0} S_{0}^{*}\right) \frac{\partial^{2} v_{2}}{\partial t^{2}}-\left(\rho_{b} I_{b}+\rho_{0} I_{0}^{*}-\frac{2 \nu_{b} I_{b} \rho_{0}}{D_{0}}\right) \frac{\partial^{4} v_{2}}{\partial t^{2} \partial x_{2}^{2}}+ \\
& {\left[E_{b} I_{b}+\left(\lambda_{0}+2 \mu_{0}\right) I_{0}^{*}-\frac{2 \nu_{b} I_{b} \tau_{0}}{D_{0}}\right] \frac{\partial^{4} v_{2}}{\partial x_{2}^{4}}-\left(T+\tau_{0} S^{*}\right) \frac{\partial^{2} v_{2}}{\partial x_{2}^{2}}+} \\
& B_{0} I_{0} \frac{\partial w_{2}}{\partial x_{2}}-\frac{\mu_{0} I_{0}^{2}}{2 \pi d^{2}}\left(v_{2}-v_{1}\right)=0 \\
& \left(\rho_{b} A_{b}+\rho_{0} S_{0}^{*}\right) \frac{\partial^{2} w_{2}}{\partial t^{2}}-\left(\rho_{b} I_{b}+\rho_{0} I_{0}^{*}-\frac{2 \nu_{b} I_{b} \rho_{0}}{D_{0}}\right) \frac{\partial^{4} w_{2}}{\partial t^{2} \partial x_{2}^{2}}+ \\
& {\left[E_{b} I_{b}+\left(\lambda_{0}+2 \mu_{0}\right) I_{0}^{*}-\frac{2 \nu_{b} I_{b} \tau_{0}}{D_{0}}\right] \frac{\partial^{4} w_{2}}{\partial x_{2}^{4}}-\left(T+\tau_{0} S^{*}\right) \frac{\partial^{2} w_{2}}{\partial x_{2}^{2}}-} \\
& B_{0} I_{0} \frac{\partial v_{2}}{\partial x_{2}}+\frac{\mu_{0} I_{0}^{2}}{2 \pi d^{2}}\left(w_{2}-w_{1}\right)=0 .
\end{aligned}
$$


To explore free vibration of the nanosystem at hand in a more general context, the following dimensionless parameters are taken into account:

$$
\begin{aligned}
& \bar{v}_{m}=\frac{v_{m}}{l_{b}}, \bar{w}_{m}=\frac{w_{m}}{l_{b}}, \xi_{m}=\frac{x_{m}}{l_{b}}, \tau=\frac{t}{l_{b}^{2}} \sqrt{\frac{E_{b} I_{b}}{\rho_{b} A_{b}}}, \lambda=\frac{l_{b}}{r_{b}}, \\
& \bar{T}=\frac{T l_{b}^{2}}{E_{b} I_{b}}, \bar{f}_{0}=\frac{B_{0} I_{0} l_{b}^{3}}{E_{b} I_{b}}, \bar{f}_{1}=\frac{\mu_{0} I_{0}^{2} l_{b}^{4}}{2 \pi d^{2} E_{b} I_{b}}, \chi_{1}=\frac{\rho_{0} S_{0}^{*}}{\rho_{b} A_{b}}, \\
& \chi_{2}=\frac{\rho_{0} I_{0}^{*}-\frac{2 \nu_{b} I_{b} \rho_{0}}{D_{0}}}{\rho_{b} I_{b}}, \chi_{3}=\frac{\left(\lambda_{0}+2 \mu_{0}\right) I_{0}^{*}-\frac{2 \nu_{b} I_{b} \tau_{0}}{D_{0}}}{E_{b} I_{b}}, \chi_{4}=\frac{\tau_{0} S_{0}^{*} l_{b}^{2}}{E_{b} I_{b}},
\end{aligned}
$$

by introducing Eq. (20) to Eqs. (19a)-(19d), the dimensionless approximate governing equations of magnetically actuated DCCNWs are obtained as:

$$
\begin{aligned}
& \left(1+\chi_{1}\right) \frac{\partial^{2} \bar{v}_{1}}{\partial \tau^{2}}-\lambda^{-2}\left(1+\chi_{2}\right) \frac{\partial^{4} \bar{v}_{1}}{\partial \tau^{2} \partial \xi_{1}^{2}}+ \\
& \left(1+\chi_{3}\right) \frac{\partial^{4} \bar{v}_{1}}{\partial \xi_{1}^{4}}-\left(\bar{T}+\chi_{4}\right) \frac{\partial^{2} \bar{v}_{1}}{\partial \xi_{1}^{2}}-\bar{f}_{0} \frac{\partial \bar{w}_{1}}{\partial \xi_{1}}+\bar{f}_{1}\left(\bar{v}_{2}-\bar{v}_{1}\right)=0 \\
& \left(1+\chi_{1}\right) \frac{\partial^{2} \bar{w}_{1}}{\partial \tau^{2}}-\lambda^{-2}\left(1+\chi_{2}\right) \frac{\partial^{4} \bar{w}_{1}}{\partial \tau^{2} \partial \xi_{1}^{2}}+ \\
& \left(1+\chi_{3}\right) \frac{\partial^{4} \bar{w}_{1}}{\partial \xi_{1}^{4}}-\left(\bar{T}+\chi_{4}\right) \frac{\partial^{2} \bar{w}_{1}}{\partial \xi_{1}^{2}}+\bar{f}_{0} \frac{\partial \bar{v}_{1}}{\partial \xi_{1}}-\bar{f}_{1}\left(\bar{w}_{2}-\bar{w}_{1}\right)=0 \\
& \left(1+\chi_{1}\right) \frac{\partial^{2} \bar{v}_{2}}{\partial \tau^{2}}-\lambda^{-2}\left(1+\chi_{2}\right) \frac{\partial^{4} \bar{v}_{2}}{\partial \tau^{2} \partial \xi_{2}^{2}}+ \\
& \left(1+\chi_{3}\right) \frac{\partial^{4} \bar{v}_{2}}{\partial \xi_{2}^{4}}-\left(\bar{T}+\chi_{4}\right) \frac{\partial^{2} \bar{v}_{2}}{\partial \xi_{2}^{2}}-\bar{f}_{0} \frac{\partial \bar{w}_{2}}{\partial \xi_{2}}-\bar{f}_{1}\left(\bar{v}_{2}-\bar{v}_{1}\right)=0 \\
& \left(1+\chi_{1}\right) \frac{\partial^{2} \bar{w}_{2}}{\partial \tau^{2}}-\lambda^{-2}\left(1+\chi_{2}\right) \frac{\partial^{4} \bar{w}_{2}}{\partial \tau^{2} \partial \xi_{2}^{2}}+ \\
& \left(1+\chi_{3}\right) \frac{\partial^{4} \bar{w}_{2}}{\partial \xi_{2}^{4}}-\left(\bar{T}+\chi_{4}\right) \frac{\partial^{2} \bar{w}_{2}}{\partial \xi_{2}^{2}}+\bar{f}_{0} \frac{\partial \bar{v}_{2}}{\partial \xi_{2}}+\bar{f}_{1}\left(\bar{w}_{2}-\bar{w}_{1}\right)=0
\end{aligned}
$$

It should be noticed that through setting $\chi_{m}=0 ; i=1,2,3,4$, Eqs. (21a)-(21d) are reduced to the equations of motion of doubly parallel current-carrying macrowires in the presence of a longitudinal magnetic field. For very lengthy nanowires (i.e., in the absence of bending rigidity and rotational inertia), the dynamic response of the nanosystem under arbitrary initial conditions was investigated by Kiani $[15,16]$. The explicit expressions of the frequencies 
and their corresponding vibration modes were obtained. The conditions lead to the dynamic instability of the nanosystem were also explained and discussed.

\subsubsection{An exact model}

By substituting Eqs. (12a) and (12b) into Eqs. (18a)-(18d), the more refined equations of motion of the magnetically actuated nanosystem are provided in the following form:

$$
\begin{aligned}
& \left(\rho_{b} A_{b}+\rho_{0} S_{0}^{*}\right) \frac{\partial^{2} v_{1}}{\partial t^{2}}-\left(\rho_{b} I_{b}+\rho_{0} I_{0}^{*}-\frac{2 \nu_{b} I_{b} \rho_{0}}{D_{0}}\right) \frac{\partial^{4} v_{1}}{\partial t^{2} \partial x_{1}^{2}}+ \\
& {\left[E_{b} I_{b}+\left(\lambda_{0}+2 \mu_{0}\right) I_{0}^{*}-\frac{2 \nu_{b} I_{b} \tau_{0}}{D_{0}}\right] \frac{\partial^{4} v_{1}}{\partial x_{1}^{4}}-\left(T+\tau_{0} S^{*}\right) \frac{\partial^{2} v_{1}}{\partial x_{1}^{2}}-} \\
& B_{0} I_{0} \frac{\partial w_{1}}{\partial x_{1}}+\frac{\mu_{0} I_{0}^{2}}{4 \pi} \int_{0}^{l_{b}} \frac{\left(x_{2}-x_{1}\right) \frac{\partial v_{2}}{\partial x_{2}}-\left(v_{2}-v_{1}\right)}{\left(\left(x_{2}-x_{1}\right)^{2}+d^{2}\right)^{\frac{3}{2}}} \mathrm{~d} x_{2}=0, \\
& \left(\rho_{b} A_{b}+\rho_{0} S_{0}^{*}\right) \frac{\partial^{2} w_{1}}{\partial t^{2}}-\left(\rho_{b} I_{b}+\rho_{0} I_{0}^{*}-\frac{2 \nu_{b} I_{b} \rho_{0}}{D_{0}}\right) \frac{\partial^{4} w_{1}}{\partial t^{2} \partial x_{1}^{2}}+ \\
& {\left[E_{b} I_{b}+\left(\lambda_{0}+2 \mu_{0}\right) I_{0}^{*}-\frac{2 \nu_{b} I_{b} \tau_{0}}{D_{0}}\right] \frac{\partial^{4} w_{1}}{\partial x_{1}^{4}}-\left(T+\tau_{0} S^{*}\right) \frac{\partial^{2} w_{1}}{\partial x_{2}^{2}}+} \\
& B_{0} I_{0} \frac{\partial v_{1}}{\partial x_{1}}+\frac{\mu_{0} I_{0}^{2}}{4 \pi} \int_{0}^{l_{b}} \frac{\left(x_{2}-x_{1}\right) \frac{\partial w_{2}}{\partial x_{2}}-\left(w_{2}-w_{1}\right)}{\left(\left(x_{2}-x_{1}\right)^{2}+d^{2}\right)^{\frac{3}{2}}} \mathrm{~d} x_{2}=0, \\
& \left(\rho_{b} A_{b}+\rho_{0} S_{0}^{*}\right) \frac{\partial^{2} v_{2}}{\partial t^{2}}-\left(\rho_{b} I_{b}+\rho_{0} I_{0}^{*}-\frac{2 \nu_{b} I_{b} \rho_{0}}{D_{0}}\right) \frac{\partial^{4} v_{2}}{\partial t^{2} \partial x_{2}^{2}}+ \\
& {\left[E_{b} I_{b}+\left(\lambda_{0}+2 \mu_{0}\right) I_{0}^{*}-\frac{2 \nu_{b} I_{b} \tau_{0}}{D_{0}}\right] \frac{\partial^{4} v_{2}}{\partial x_{2}^{4}}-\left(T+\tau_{0} S^{*}\right) \frac{\partial^{2} v_{2}}{\partial x_{2}^{2}}-} \\
& B_{0} I_{0} \frac{\partial w_{2}}{\partial x_{2}}-\frac{\mu_{0} I_{0}^{2}}{4 \pi} \int_{0}^{l_{b}} \frac{\left(x_{2}-x_{1}\right) \frac{\partial v_{1}}{\partial x_{1}}-\left(v_{2}-v_{1}\right)}{\left(\left(x_{2}-x_{1}\right)^{2}+d^{2}\right)^{\frac{3}{2}}} \mathrm{~d} x_{1}=0 \text {, } \\
& \left(\rho_{b} A_{b}+\rho_{0} S_{0}^{*}\right) \frac{\partial^{2} w_{2}}{\partial t^{2}}-\left(\rho_{b} I_{b}+\rho_{0} I_{0}^{*}-\frac{2 \nu_{b} I_{b} \rho_{0}}{D_{0}}\right) \frac{\partial^{4} w_{2}}{\partial t^{2} \partial x_{2}^{2}}+ \\
& {\left[E_{b} I_{b}+\left(\lambda_{0}+2 \mu_{0}\right) I_{0}^{*}-\frac{2 \nu_{b} I_{b} \tau_{0}}{D_{0}}\right] \frac{\partial^{4} w_{2}}{\partial x_{2}^{4}}-\left(T+\tau_{0} S^{*}\right) \frac{\partial^{2} w_{2}}{\partial x_{2}^{2}}+} \\
& B_{0} I_{0} \frac{\partial v_{2}}{\partial x_{2}}-\frac{\mu_{0} I_{0}^{2}}{4 \pi} \int_{0}^{l_{b}} \frac{\left(x_{2}-x_{1}\right) \frac{\partial w_{1}}{\partial x_{1}}-\left(w_{2}-w_{1}\right)}{\left(\left(x_{2}-x_{1}\right)^{2}+d^{2}\right)^{\frac{3}{2}}} \mathrm{~d} x_{1}=0 .
\end{aligned}
$$


Finally, by introducing the dimensionless parameters in Eq. (20) to Eqs. (22a)-(22d), the dimensionless equations of motion of magnetically actuated DCCNWs based on the proposed refined model are extracted as:

$$
\begin{aligned}
& \left(1+\chi_{1}\right) \frac{\partial^{2} \bar{v}_{1}}{\partial \tau^{2}}-\lambda^{-2}\left(1+\chi_{2}\right) \frac{\partial^{4} \bar{v}_{1}}{\partial \tau^{2} \partial \xi_{1}^{2}}+\left(1+\chi_{3}\right) \frac{\partial^{4} \bar{v}_{1}}{\partial \xi_{1}^{4}}- \\
& \left(\bar{T}+\chi_{4}\right) \frac{\partial^{2} \bar{v}_{1}}{\partial \xi_{1}^{2}}-\bar{f}_{0} \frac{\partial \bar{w}_{1}}{\partial \xi_{1}}+\frac{\bar{f}_{1} \bar{d}^{2}}{2} \int_{0}^{1} \frac{\left(\xi_{2}-\xi_{1}\right) \frac{\partial \bar{v}_{2}}{\partial \xi_{2}}-\left(\bar{v}_{2}-\bar{v}_{1}\right)}{\left(\left(\xi_{2}-\xi_{1}\right)^{2}+\bar{d}^{2}\right)^{\frac{3}{2}}} \mathrm{~d} \xi_{2}=0 \\
& \left(1+\chi_{1}\right) \frac{\partial^{2} \bar{w}_{1}}{\partial \tau^{2}}-\lambda^{-2}\left(1+\chi_{2}\right) \frac{\partial^{4} \bar{w}_{1}}{\partial \tau^{2} \partial \xi_{1}^{2}}+\left(1+\chi_{3}\right) \frac{\partial^{4} \bar{w}_{1}}{\partial \xi_{1}^{4}}- \\
& \left(\bar{T}+\chi_{4}\right) \frac{\partial^{2} \bar{w}_{1}}{\partial \xi_{1}^{2}}+\bar{f}_{0} \frac{\partial \bar{v}_{1}}{\partial \xi_{1}}+\frac{\bar{f}_{1} \bar{d}^{2}}{2} \int_{0}^{1} \frac{\left(\xi_{2}-\xi_{1}\right) \frac{\partial \bar{w}_{2}}{\partial \xi_{2}}-\left(\bar{w}_{2}-\bar{w}_{1}\right)}{\left(\left(\xi_{2}-\xi_{1}\right)^{2}+\bar{d}^{2}\right)^{\frac{3}{2}}} \mathrm{~d} \xi_{2}=0 \\
& \left(1+\chi_{1}\right) \frac{\partial^{2} \bar{v}_{2}}{\partial \tau^{2}}-\lambda^{-2}\left(1+\chi_{2}\right) \frac{\partial^{4} \bar{v}_{2}}{\partial \tau^{2} \partial \xi_{2}^{2}}+\left(1+\chi_{3}\right) \frac{\partial^{4} \bar{v}_{2}}{\partial \xi_{2}^{4}}- \\
& \left(\bar{T}+\chi_{4}\right) \frac{\partial^{2} \bar{v}_{2}}{\partial \xi_{2}^{2}}-\bar{f}_{0} \frac{\partial \bar{w}_{2}}{\partial \xi_{2}}-\frac{\bar{f}_{1} \bar{d}^{2}}{2} \int_{0}^{1} \frac{\left(\xi_{2}-\xi_{1}\right) \frac{\partial \bar{v}_{1}}{\partial \xi_{1}}-\left(\bar{v}_{2}-\bar{v}_{1}\right)}{\left(\left(\xi_{2}-\xi_{1}\right)^{2}+\bar{d}^{2}\right)^{\frac{3}{2}}} \mathrm{~d} \xi_{1}=0 \\
& \left(1+\chi_{1}\right) \frac{\partial^{2} \bar{w}_{2}}{\partial \tau^{2}}-\lambda^{-2}\left(1+\chi_{2}\right) \frac{\partial^{4} \bar{w}_{2}}{\partial \tau^{2} \partial \xi_{2}^{2}}+\left(1+\chi_{3}\right) \frac{\partial^{4} \bar{w}_{2}}{\partial \xi_{2}^{4}}- \\
& \left(\bar{T}+\chi_{4}\right) \frac{\partial^{2} \bar{w}_{2}}{\partial \xi_{2}^{2}}+\bar{f}_{0} \frac{\partial \bar{v}_{2}}{\partial \xi_{2}}-\frac{\bar{f}_{1} \bar{d}^{2}}{2} \int_{0}^{1} \frac{\left(\xi_{2}-\xi_{1}\right) \frac{\partial \bar{w}_{1}}{\partial \xi_{1}}-\left(\bar{w}_{2}-\bar{w}_{1}\right)}{\left(\left(\xi_{2}-\xi_{1}\right)^{2}+\bar{d}^{2}\right)^{\frac{3}{2}}} \mathrm{~d} \xi_{1}=0 .
\end{aligned}
$$

Eqs. (23a)-(23d) represent four coupled integro-partial differential equations (IPDE) that should be appropriately solved. In the following part, free vibration of the nanosystem are examined by employing AMM.

\subsection{Dynamic analysis of the magnetically affected nanosystem via AMM}

\subsubsection{Free transverse vibration of the nanosystem using the proposed approximate model}

Let express the dimensionless transverse displacements of the magnetically actuated nanosystem as follows:

$$
\bar{v}_{m}\left(\xi_{m}, \tau\right)=\sum_{i=1}^{N M_{m}} \phi_{i}^{v_{m}}\left(\xi_{m}\right) \bar{v}_{m_{i}}(\tau), \bar{w}\left(\xi_{m}, \tau\right)=\sum_{i=1}^{N M_{m}} \phi_{i}^{w_{m}}\left(\xi_{m}\right) \bar{w}_{m_{i}}(\tau) ; m=1,2,
$$


where $N M_{m}$ is the number of considered modes pertinent to the $m$ th NW, $\phi_{i}^{v_{m}}(\xi)$ and $\phi_{i}^{w_{m}}(\xi)$ are the $i$ th mode shape functions associated with $\bar{v}_{m}$ and $\bar{w}_{m}, \bar{v}_{m_{i}}(\tau)$ and $\bar{w}_{m_{i}}(\tau)$ are their corresponding time-dependent parameters. Now we premultiply both sides of Eqs. (21a)(21d) in order by $\delta \bar{v}_{1}, \delta \bar{w}_{1}, \delta \bar{v}_{2}$, and $\delta \bar{w}_{2}$, where $\delta$ is the variational sign. By taking the integral from the resulting expressions, and taking the required integration by parts, it is obtained:

$$
\begin{gathered}
{\left[\begin{array}{cccc}
{\left[\overline{\mathbf{M}}_{b}\right]^{v_{1} v_{1}}} & {\left[\overline{\mathbf{M}}_{b}\right]^{v_{1} w_{1}}} & {\left[\overline{\mathbf{M}}_{b}\right]^{v_{1} v_{2}}} & {\left[\overline{\mathbf{M}}_{b}\right]^{v_{1} w_{2}}} \\
{\left[\overline{\mathbf{M}}_{b}\right]^{w_{1} v_{1}}} & {\left[\overline{\mathbf{M}}_{b}\right]^{w_{1} w_{1}}} & {\left[\overline{\mathbf{M}}_{b}\right]^{w_{1} v_{2}}} & {\left[\overline{\mathbf{M}}_{b}\right]^{w_{1} w_{2}}} \\
{\left[\overline{\mathbf{M}}_{b}\right]^{v_{2} v_{1}}} & {\left[\overline{\mathbf{M}}_{b}\right]^{v_{2} w_{1}}} & {\left[\overline{\mathbf{M}}_{b}\right]^{v_{2} v_{2}}} & {\left[\overline{\mathbf{M}}_{b}\right]^{v_{2} w_{2}}} \\
{\left[\overline{\mathbf{M}}_{b}\right]^{w_{2} v_{1}}} & {\left[\overline{\mathbf{M}}_{b}\right]^{w_{2} w_{1}}} & {\left[\overline{\mathbf{M}}_{b}\right]^{w_{2} v_{2}}} & {\left[\overline{\mathbf{M}}_{b}\right]^{w_{2} w_{2}}}
\end{array}\right]\left\{\begin{array}{c}
\frac{\mathrm{d}^{2} \overline{\mathbf{v}}_{1}}{\mathrm{~d} \tau^{2}} \\
\frac{\mathrm{d}^{2} \overline{\mathbf{w}}_{1}}{\mathrm{~d} \tau^{2}} \\
\frac{\mathrm{d}^{2} \overline{\mathbf{v}}_{2}}{\mathrm{~d} \tau^{2}} \\
\frac{\mathrm{d}^{2} \overline{\mathbf{w}}_{2}}{\mathrm{~d} \tau^{2}}
\end{array}\right\}+} \\
{\left[\begin{array}{cccc}
{\left[\overline{\mathbf{K}}_{b}\right]^{v_{1} v_{1}}} & {\left[\overline{\mathbf{K}}_{b}\right]^{v_{1} w_{1}}} & {\left[\overline{\mathbf{K}}_{b}\right]^{v_{1} v_{2}}} & {\left[\overline{\mathbf{K}}_{b}\right]^{v_{1} w_{2}}} \\
{\left[\overline{\mathbf{K}}_{b}\right]^{w_{1} v_{1}}} & {\left[\overline{\mathbf{K}}_{b}\right]^{w_{1} w_{1}}} & {\left[\overline{\mathbf{K}}_{b}\right]^{w_{1} v_{2}}} & {\left[\overline{\mathbf{K}}_{b}\right]^{w_{1} w_{2}}} \\
{\left[\overline{\mathbf{K}}_{b}\right]^{v_{2} v_{1}}} & {\left[\overline{\mathbf{K}}_{b}\right]^{v_{2} w_{1}}} & {\left[\overline{\mathbf{K}}_{b}\right]^{v_{2} v_{2}}} & {\left[\overline{\mathbf{K}}_{b}\right]^{v_{2} w_{2}}} \\
{\left[\overline{\mathbf{K}}_{b}\right]^{w_{2} v_{1}}} & {\left[\overline{\mathbf{K}}_{b}\right]^{w_{2} w_{1}}} & {\left[\overline{\mathbf{K}}_{b}\right]^{w_{2} v_{2}}} & {\left[\overline{\mathbf{K}}_{b}\right]^{w_{2} w_{2}}}
\end{array}\right]\left\{\begin{array}{c}
\overline{\mathbf{v}}_{1} \\
\overline{\mathbf{w}}_{1} \\
\overline{\mathbf{v}}_{2} \\
\overline{\mathbf{w}}_{2}
\end{array}\right\}=\left\{\begin{array}{c}
\mathbf{0} \\
\mathbf{0} \\
\mathbf{0} \\
\mathbf{0}
\end{array}\right\},}
\end{gathered}
$$

where the nonzero submatrices in Eq. (25) are defined by:

$$
\begin{gathered}
{\left[\overline{\mathbf{M}}_{b}\right]_{i j}^{v_{m} v_{m}}=\int_{0}^{1}\left(\left(1+\chi_{1}\right) \phi_{i}^{v_{m}} \phi_{j}^{v_{m}}+\lambda^{-2}\left(1+\chi_{2}\right) \frac{\mathrm{d} \phi_{i}^{v_{m}}}{\mathrm{~d} \xi_{m}} \frac{\mathrm{d} \phi_{j}^{v_{m}}}{\mathrm{~d} \xi_{m}}\right) \mathrm{d} \xi_{m}} \\
{\left[\overline{\mathbf{M}}_{b}\right]_{i j}^{w_{m} w_{m}}=\int_{0}^{1}\left(\left(1+\chi_{1}\right) \phi_{i}^{w_{m}} \phi_{j}^{w_{m}}+\lambda^{-2}\left(1+\chi_{2}\right) \frac{\mathrm{d} \phi_{i}^{w_{m}}}{\mathrm{~d} \xi_{m}} \frac{\mathrm{d} \phi_{j}^{w_{m}}}{\mathrm{~d} \xi_{m}}\right) \mathrm{d} \xi_{m}} \\
{\left[\overline{\mathbf{K}}_{b}\right]_{i j}^{v_{m} v_{m}}=\int_{0}^{1}\left(\left(1+\chi_{3}\right) \frac{\mathrm{d}^{2} \phi_{i}^{v_{m}}}{\mathrm{~d} \xi_{m}^{2}} \frac{\mathrm{d}^{2} \phi_{j}^{v_{m}}}{\mathrm{~d} \xi_{m}^{2}}+\left(\bar{T}+\chi_{4}\right) \frac{\mathrm{d} \phi_{i}^{v_{m}}}{\mathrm{~d} \xi_{m}} \frac{\mathrm{d} \phi_{j}^{v_{m}}}{\mathrm{~d} \xi_{m}}-\bar{f}_{1} \phi_{i}^{v_{m}} \phi_{j}^{v_{m}}\right) \mathrm{d} \xi_{m}} \\
{\left[\overline{\mathbf{K}}_{b}\right]_{i j}^{v_{m} w_{m}}=-\int_{0}^{1} \bar{f}_{0} \phi_{i}^{v_{m}} \frac{\mathrm{d} \phi_{j}^{w_{m}}}{\mathrm{~d} \xi_{m}} \mathrm{~d} \xi_{m}} \\
{\left[\overline{\mathbf{K}}_{b}\right]_{i j}^{v_{m} v_{n}}=\int_{0}^{1} \bar{f}_{1} \phi_{i}^{v_{m}} \phi_{j}^{v_{n}} \mathrm{~d} \xi_{n} ; m \neq n}
\end{gathered}
$$




$$
\begin{gathered}
{\left[\overline{\mathbf{K}}_{b}\right]_{i j}^{w_{m} v_{m}}=\int_{0}^{1} \bar{f}_{0} \phi_{i}^{w_{m}} \frac{\mathrm{d} \phi_{j}^{v_{m}}}{\mathrm{~d} \xi_{m}} \mathrm{~d} \xi_{m}} \\
{\left[\overline{\mathbf{K}}_{b}\right]_{i j}^{w_{m} w_{m}}=\int_{0}^{1}\left(\left(1+\chi_{3}\right) \frac{\mathrm{d}^{2} \phi_{i}^{w_{m}}}{\mathrm{~d} \xi_{m}^{2}} \frac{\mathrm{d}^{2} \phi_{j}^{w_{m}}}{\mathrm{~d} \xi_{m}^{2}}+\left(\bar{T}+\chi_{4}\right) \frac{\mathrm{d} \phi_{i}^{w_{m}}}{\mathrm{~d} \xi_{m}} \frac{\mathrm{d} \phi_{j}^{w_{n}}}{\mathrm{~d} \xi_{m}}+\bar{f}_{1} \phi_{i}^{w_{m}} \phi_{j}^{w_{m}}\right) \mathrm{d} \xi_{m},(2} \\
{\left[\overline{\mathbf{K}}_{b}\right]_{i j}^{w_{m} w_{n}}=-\int_{0}^{1} \bar{f}_{1} \phi_{i}^{w_{m}} \phi_{j}^{w_{n}} \mathrm{~d} \xi_{n} ; m \neq n} \\
\overline{\mathbf{v}}_{m}(\tau)=<\bar{v}_{i_{1}}(\tau), \bar{v}_{i_{2}}(\tau), \ldots, \bar{v}_{i_{N M_{m}}}(\tau)>^{\mathrm{T}} \\
\overline{\mathbf{w}}_{m}(\tau)=<\bar{w}_{i_{1}}(\tau), \bar{w}_{i_{2}}(\tau), \ldots, \bar{w}_{i_{N M_{m}}}(\tau)>^{\mathrm{T}}
\end{gathered}
$$

where $m, n=1,2$. For frequency analysis of the problem, let: $\overline{\mathbf{v}}_{m}(\tau)=\overline{\mathbf{v}}_{m 0} e^{\mathrm{i} \varpi \tau}$ and $\overline{\mathbf{w}}_{m}(\tau)=$ $\overline{\mathbf{w}}_{m 0} e^{\mathrm{i} \varpi \tau}$ where $\varpi$ represents the dimensionless frequency, $\mathrm{i}=\sqrt{-1}$, and $\overline{\mathbf{v}}_{m 0}$ and $\overline{\mathbf{w}}_{m 0}$ are the dimensionless amplitude vectors. By substituting these expressions into Eq. (25), and by solving the resulting set of eigenvalue equations, the eigenvalues and their corresponding mode shapes could be readily evaluated.

\subsubsection{Free transverse vibration of the nanosystem using the proposed exact model}

To construct the discretized version of the governing equations via the suggested exact formulations, we premultiply both sides of Eqs. (23a)-(23d) by $\delta \bar{v}_{1}, \delta \bar{w}_{1}, \delta \bar{v}_{2}$, and $\delta \bar{w}_{2}$, respectively. By integration of the resulting statements over the dimensionless domains of the NWs, taking the required integration by parts, and introducing Eq. (24) to the obtained expressions, a set of ordinary equations of motion similar to those in Eq. (25) is derived. All dimensionless mass submatrices and some stiffness submatrices are identical to those of the approximate model whereas most of the dimensionless stiffness submatrices are modified as follows:

$$
\begin{aligned}
{\left[\overline{\mathbf{K}}_{b}\right]_{i j}^{v_{m} v_{m}}=} & \int_{0}^{1}\left(\left(1+\chi_{3}\right) \frac{\mathrm{d}^{2} \phi_{i}^{v_{m}}}{\mathrm{~d} \xi_{m}^{2}} \frac{\mathrm{d}^{2} \phi_{j}^{v_{m}}}{\mathrm{~d} \xi_{m}^{2}}+\left(\bar{T}+\chi_{4}\right) \frac{\mathrm{d} \phi_{i}^{v_{m}}}{\mathrm{~d} \xi_{m}} \frac{\mathrm{d} \phi_{j}^{v_{m}}}{\mathrm{~d} \xi_{m}}\right) \mathrm{d} \xi_{m} \\
& +\frac{\bar{f}_{1} \bar{d}^{2}}{2} \int_{0}^{1} \int_{0}^{1} \frac{\phi_{i}^{v_{m}}\left(\xi_{m}\right) \phi_{j}^{v_{m}}\left(\xi_{m}\right)}{\left(\left(\xi_{2}-\xi_{1}\right)^{2}+\bar{d}^{2}\right)^{\frac{3}{2}}} \mathrm{~d} \xi_{2} \mathrm{~d} \xi_{1}
\end{aligned}
$$




$$
\begin{aligned}
{\left[\overline{\mathbf{K}}_{b}\right]_{i j}^{v_{m} v_{n}}=} & -\frac{\bar{f}_{1} \bar{d}^{2}}{2} \int_{0}^{1} \int_{0}^{1} \frac{\phi_{i}^{v_{m}}\left(\left(\xi_{m}-\xi_{n}\right) \frac{\mathrm{d} \phi_{j}^{v_{n}}}{\mathrm{~d} \xi_{n}}+\phi_{j}^{v_{n}}\right)}{\left(\left(\xi_{2}-\xi_{1}\right)^{2}+\bar{d}^{2}\right)^{\frac{3}{2}}} \mathrm{~d} \xi_{m} \mathrm{~d} \xi_{n} ; m \neq n, \\
{\left[\overline{\mathbf{K}}_{b}\right]_{i j}^{w_{m} w_{m}}=} & \int_{0}^{1}\left(\left(1+\chi_{3}\right) \frac{\mathrm{d}^{2} \phi_{i}^{w_{m}}}{\mathrm{~d} \xi_{m}^{2}} \frac{\mathrm{d}^{2} \phi_{j}^{w_{m}}}{\mathrm{~d} \xi_{m}^{2}}+\left(\bar{T}+\chi_{4}\right) \frac{\mathrm{d} \phi_{i}^{w_{m}}}{\mathrm{~d} \xi_{m}} \frac{\mathrm{d} \phi_{j}^{w_{m}}}{\mathrm{~d} \xi_{m}}\right) \mathrm{d} \xi_{m}+ \\
& +\frac{\bar{f}_{1} \bar{d}^{2}}{2} \int_{0}^{1} \int_{0}^{1} \frac{\phi_{i}^{w_{m}}\left(\xi_{m}\right) \phi_{j}^{w_{m}}\left(\xi_{m}\right)}{\left(\left(\xi_{2}-\xi_{1}\right)^{2}+\bar{d}^{2}\right)^{\frac{3}{2}} \mathrm{~d} \xi_{2} \mathrm{~d} \xi_{1},} \\
{\left[\overline{\mathbf{K}}_{b}\right]_{i j}^{w_{m} w_{n}}=} & -\frac{\bar{f}_{1} \bar{d}^{2}}{2} \int_{0}^{1} \int_{0}^{1} \frac{\phi_{i}^{w_{m}}\left(\left(\xi_{m}-\xi_{n}\right) \frac{\mathrm{d} \phi_{j}^{w_{n}}}{\mathrm{~d} \xi_{n}}+\phi_{j}^{w_{n}}\right)}{\left(\left(\xi_{2}-\xi_{1}\right)^{2}+\bar{d}^{2}\right)^{\frac{3}{2}}} \mathrm{~d} \xi_{m} \mathrm{~d} \xi_{n} ; m \neq n,
\end{aligned}
$$

where $m, n=1,2$. By following the given procedure in the previous part, the frequencies and the vibration modes of the nanosystem based on the newly developed exact model could be easily obtained.

\section{Results and discussion}

In this part, the capabilities of the approximate model in predicting the natural frequencies of the nanosystem based on the exact model are explained. Additionally, the role of the surface energy effect on the obtained results is displayed for different levels of the electric current, magnetic field strength, and slenderness ratio. For this purpose, consider DCCNWs made from Au with the following bulk and surface properties: $E_{b}=37 \times 10^{9}, \nu_{b}=0.26$, $\rho_{b}=19300 \mathrm{~kg} / \mathrm{m}^{3}, \tau_{0}=1.4 \mathrm{~N} / \mathrm{m}, E_{0}=-3.6 \mathrm{~N} / \mathrm{m}, \lambda_{0}=3.6 \mathrm{~N} / \mathrm{m}$, and $\rho_{0}=1.5 \times 10^{-7} \mathrm{~kg} / \mathrm{m}^{2}$. Free vibration of the nanosystem for two kinds of end conditions are of interest: nanowires with simply supported (SS) ends and clamped-clamped (CC) condition. In numerical analysis of the problem, the following admissible mode shapes are taken into account:

$$
\text { SS condition: } \phi_{i}^{v_{m}}(\xi)=\phi_{i}^{w_{m}}(\xi)=\sin (i \pi \xi) ; m=1,2
$$




$$
\begin{aligned}
& \text { CC condition: } \phi_{i}^{v_{m}}(\xi)=\phi_{i}^{w_{m}}(\xi)= \\
& \sin \left(e_{i} \xi\right) /\left(\sin \left(e_{i}\right)-\sinh \left(e_{i}\right)\right)+\cos \left(e_{i} \xi\right) /\left(\cos \left(e_{i}\right)-\cosh \left(e_{i}\right)\right) \\
& -\sinh \left(e_{i} \xi\right) /\left(\sin \left(e_{i}\right)-\sinh \left(e_{i}\right)\right)-\cosh \left(e_{i} \xi\right) /\left(\cos \left(e_{i}\right)-\cosh \left(e_{i}\right)\right)
\end{aligned}
$$

where

$$
e_{1}=4.730, e_{2}=7.853, e_{i}=\frac{(2 i+1) \pi}{2} ; i \geq 3
$$

\subsection{The capabilities of the proposed exact model}

In the previous works $[12,13]$, the NWs' length was large enough that the approximate model could be reasonably implemented for transverse vibrations of the nanosystem. Because of this fact, the bending rigidity of the NWs was also ignored. Herein, a more accurate model is developed by including the bending rigidity of both the bulk and the surface layer. It implies that the previously developed models for the problem of current-carrying doublenanowire-systems immersed in a longitudinal magnetic field is basically modified accounting for both the bending rigidity and the interwire magnetic forces. Therefore, it is expected that the given model would expand our view to some unseen realms of the previous models. For this purpose, the obtained results by the exact model are compared with those of the approximate model.

In Figs. 2(a) and 2(b), the approximate and exact fundamental frequencies of the nanosystem in terms of the interwire distance have been plotted. The results are demonstrated for SS and CC nanosystems with three levels of the electric current whereas the strength of the applied longitudinal magnetic field and the slenderness ratio of the NWs are constant. As it is obvious in Fig. 2, both the approximate model and the exact model predict that the fundamental frequency would reduce as the interwire distance decreases. Commonly, the rate of reduction of the plots based on the approximate analysis is more apparent with respect to that of the exact model, particularly for higher levels of the electric currents. Based on a close survey of the plotted results, it is indicated that the relative discrepancies between the predicted fundamental frequencies by the approximate model and 
those of the exact model would lessen as the interwire distance increases. Such a fact is attributed to this reason that the interwire interactional forces would diminish as the interwire distance grow, and the role of the longitudinal magnetic field on the vibration of the nanosystem becomes important. Additionally, the above-mentioned relative discrepancies would increase by increasing of the electric current. For instance, the approximate model can capture the predicted fundamental frequencies of the $\mathrm{SS}(\mathrm{CC})$ nanosystem carrying electric currents $\bar{I}=0.1,1$, and 2 with relative errors lower than $0.03(0.007), 3.8(0.75)$, and $12.4(1.8)$ percent, respectively. This data also reveals that the discrepancies between the obtained results by the approximate model and those of the exact model for nanosystems with SS ends are generally lower with respect to the nanosystems with $\mathrm{CC}$ ends. Both models also display that the variation of the interwire distance is more influential on the variation of the fundamental frequency of SS nanosystems in compare to the CC nanosystems.

We are also interested in understanding that how variation of the slenderness ratio would influence on the efficiency of the approximate model. To this end, the predicted fundamental frequencies of the nanosystems with SS and CC ends as a function of the slenderness ratio have been plotted in Figs. 3(a) and 3(b) for three levels of the electric current. The dimensionless electric current and magnetic field strength are defined such that the variation of the slenderness ratio would have no influence on the variation of the electric current and the magnetic field strength. For a given electric current intensity and all considered boundary conditions, the relative discrepancies between the plotted results based on the exact model and those of the approximate model would grow as the slenderness ratio increases. Such an issue is more apparent for higher levels of the electric current, particularly in the case of SS boundary condition. For a nanosystem with $\mathrm{SS}(\mathrm{CC})$ boundary condition and $\lambda=30$, the above-mentioned discrepancies for the cases of $\bar{I}_{0}^{*}=0.1,1$, and 2 in order are about $0.05(0.012), 6.2(1.56)$, and 24.3(3.94) percent. In all demonstrated plots, the nanosystem based on the exact model is stiffer than that based on the approximate model. The main reason of this fact is that the approximate formulation of the Lorentz magnetic force 
has been constructed based on this important assumption that the length of the NWs is infinite. It means that the dynamic interactional forces between double current-carrying NWs are overestimated by the approximate model, and thereby, the transverse stiffness of the nanosystem would be underestimated with respect to the real situation. As a result, the predicted frequencies by the approximate model would be generally lower than those obtained via the exact model.

\subsection{The effect of the surface energy on free vibration of the nanosystem}

A very exciting study has been carried out to determine the role of the surface energy on vibrational behavior of the nanosystem. In Figs. 4(a) and 4(b), the plots of the fundamental frequency in terms of the electric current have been provided for a constant magnetic field strength. The results have been provided for both SS and CC nanosystems with three levels of the slenderness ratio (i.e., $\lambda=15,20$, and 25 ). The obtained results by considering the surface energy effect and without that have been presented by the solid lines and dashed lines, respectively. Irrespective of the surface energy effect, the fundamental frequencies of the nanosystems with SS and CC ends reduce as the electric current increases. Such a reduction is more obvious for SS nanosystems with higher slenderness ratio. For the nanosystem with $\lambda=15$ under SS boundary condition, the predicted fundamental frequencies by considering the surface effect are overestimated by those predicted by the classical continuum theory. However, in the cases of $\lambda=20$ and 25 , the obtained fundamental frequencies based on the surface elasticity theory are greater than those obtained via the classical continuum theory for the considered range of the electric current. Furthermore, for CC nanosystems and all considered slenderness ratios, the predicted fundamental frequencies by the surface elasticity theory are lower than those of the classical continuum theory. Such a complex behavior of the nanosystem due to the inclusion of the surface energy effect is mainly related to the positive or negative contributions of the Young's modulus (i.e., $E_{0}=\lambda_{0}+2 \mu_{0}$ ) and the residual surface stress of the surface layer into the transverse stiffness expressions. For 
the considered gold nanowires, $\tau_{0}>0$ while $E_{0}<0$. It implies that the residual surface stress positively contributes to the lateral stiffness and thereby, the natural frequencies of the nanosystem increase while the elastic modulus of the surface layer reduces the lateral stiffness of the nanosystem (see Eqs. 28(c) and 28(g)). A combination of these effects leads to a decrease or an increase of the natural frequencies depends on the length (i.e., slenderness ratio) of the NWs and their boundary conditions (i.e., in which directly affects on the mode shapes). Regarding nanosystems with CC boundary conditions, a detailed investigation of the plotted results shows that the role of the surface energy on the vibration behavior of the nanosystem increases with the electric current. Such a fact is more obvious for more slender nanosystems; however, the surface effect becomes diminish as the slenderness ratio of the nanosystem increases. Concerning SS nanosystems, variation of the surface energy effect in terms of the electric current also depends on the slenderness ratio. Generally, the relative discrepancies between the fundamental frequencies based on the surface elasticity theory and those of the classical continuum theory would increase by increasing of the electric current. Similar to individual current-carrying nanowires in a longitudinal magnetic field, it is expected that the relative discrepancy between the above-mentioned theories reaches to its maximum value at the critical electric current (i.e., the electric current corresponds to the zero fundamental frequency).

In Figs. 5(a) and 5(b), the plots of the fundamental frequencies of SS and CC nanosystems as a function of the magnetic field strength are provided for three levels of the slenderness ratio. A close survey of the plotted results in Figs. 5(a) and 5(b) reveals that the effect of the magnetic field strength on the vibration behavior of the nanosystems is identical to that of the electric current in which comprehensively explained in the previous part. For the sake of brevity, such explanations are not given. 


\section{Conclusions}

Free transverse vibrations of magnetically actuated DCCNWs are studied in the context of theory of surface elasticity of the Gurtin-Murdoch. Based on the Biot-Savart law and Lorentz law, two sets of formulations are developed: approximate and exact versions of the interactional magnetic forces on the CCNWs. By exploiting the Rayleigh beam theory, the equations of motion of the nanosystem are derived accounting for the surface energy effect. Based on the approximate magnetic force, the derived governing equations are four coupled PDEs whereas those obtained via the exact formulations are four coupled IPDEs. These equations are solved via AMM for nanosystems with simple and clamped ends. The capabilities of the approximate model in capturing the predicted fundamental frequency by the exact model are displayed. The influences of the slenderness ratio, interwire distance, electric current, magnetic field strength, and surface energy effect on the free vibration behavior of the nanosystem are investigated. The major obtained results are as:

1. By increasing of the electric current or magnetic field strength, the discrepancy between the predicted fundamental frequency by the exact model and that of the approximate model would grow. Additionally, the exact model predicts that the nanosystem would be commonly stiffer with respect to that based on the approximate model. Such a fact is more obvious for more slender nanosystems with lower interwire distances.

2. As the constraints of the nanosystem at its supports increase, the discrepancies between the results obtained based on the exact model and those of the approximate model would generally reduce. According to the demonstrated fundamental frequencies based on these models, these discrepancies for fully clamped nanosystems are apparently lower than those of the simply supported nanosystems.

3. Generally, the role of the surface energy effect on the free dynamic response of the nanosystem becomes more obvious as the electric current or magnetic field strength increases. This issue is more apparent for nanosystems with higher slenderness ratios. In fact, variation of the electric current or magnetic field strength has a more influence on the free vibration 
of higher slender nanosystems.

\section{Acknowledgement}

The financial supports of Iran National Science Foundation (INSF), research department of K.N. Toosi University of Technology, and Iran Nanotechnology Initiative Council is gratefully acknowledged. 


\section{References}

[1] C.M. Lieber, The incredible shrinking circuit, Sci. Am. 285 (2001) 58.

[2] Y. Cui, C.M. Lieber, Functional nanoscale electronic devices assembled using silicon nanowire building blocks, Science 291 (2001) 851-853.

[3] Z.L. Wang, J. Song, Piezoelectric nanogenerators based on zinc oxide nanowire arrays, Science 312 (2006) 242-246.

[4] X. Wang, J. Song, J. Liu, Z.L. Wang, Direct-current nanogenerator driven by ultrasonic waves, Science 316 (2007) 102-105.

[5] Y. Qin, X. Wang, Z.L. Wang, Microfibre-nanowire hybrid structure for energy scavenging, Nature 451 (2008) 809-813.

[6] M.S. Gudiksen, L. Lauhon, J. Wang, D.C. Smith, C.M. Lieber, Growth of nanowire superlattice structures for nanoscale photonics and electronics, Nature 415 (2002) 617620.

[7] M.C. McAlpine, R.S. Friedman, S. Jin, K.H. Lin, W.U. Wang, C.M. Lieber, Highperformance nanowire electronics and photonics on glass and plastic substrates, Nano Lett. 3 (2003) 1531-1535.

[8] C.J. Barrelet, A.B. Greytak, C.M. Lieber, Nanowire photonic circuit elements, Nano Lett. 4 (2004) 1981-1985.

[9] G. Zheng, F. Patolsky, Y. Cui, W.U. Wang, C.M. Lieber, Multiplexed electrical detection of cancer markers with nanowire sensor arrays, Nat. Biotechnol. 23 (2005) 1294-1301.

[10] F. Patolsky, C.M. Lieber, Nanowire nanosensors, Mater. Today 8 (2005) 20-28.

[11] X. Wang, J. Zhou, J. Song, J. Liu, N. Xu, Z.L. Wang, Piezoelectric field effect transistor and nanoforce sensor based on a single ZnO nanowire, Nano Lett. 6 (2006) 2768-2772.

[12] K. Kiani, Surface effect on free transverse vibrations and dynamic instability of currentcarrying nanowires in the presence of a longitudinal magnetic field, Phys. Lett. A 378 (2014) 1834-1840. 
[13] K. Kiani, Forced vibrations of a current-carrying nanowire in a longitudinal magnetic field accounting for both surface energy and size effects, Phys. E 63 (2014) 27-35.

[14] K. Kiani, Vibrations and instability of pretensioned current-carrying nanowires acted upon by a suddenly applied three-dimensional magnetic field, Mater. Chem. Phys. 162 (2015) 531-541.

[15] K. Kiani, Vibrations and instability of double-nanowire-systems as electric current carriers, Mod. Phys. Lett. B 29 (2015) 1550144.

[16] K. Kiani, Stability and vibrations of doubly parallel current-carrying nanowires immersed in a longitudinal magnetic field, Phys. Lett. A 379 (2015) 348-360.

[17] M.E. Gurtin, A.I. Murdoch, A continuum theory of elastic material surfaces, Arch. Ration. Mech. An. 57 (1975) 291-323.

[18] M.E. Gurtin, A.I. Murdoch, Effect of surface stress on wave propagation in solids, J. Appl. Phys. 47 (1976) 4414-4421.

[19] M.E. Gurtin, A.I. Murdoch, Surface stress in solids, Int. J. Solids Struct. 14 (1978) 431-440.

[20] J. He, C.M. Lilley, Surface stress effect on bending resonance of nanowires with different boundary conditions, Appl. Phys. Lett. 93 (2008) 263108.

[21] F. Song, G.L. Huang, Modeling of surface stress effects on bending behavior of nanowires: Incremental deformation theory, Phys. Lett. A 373 (2009) 3969-3973.

[22] L.Y. Jiang, Z. Yan, Timoshenko beam model for static bending of nanowires with surface effects, Phys. E 42 (2010) 2274-2279.

[23] Q. Wu, A.A. Volinsky, L. Qiao, Y. Su, Surface effects on static bending of nanowires based on non-local elasticity theory, Prog. Nat. Sci. 25 (2015) 520-524.

[24] F.F. Mahmoud, M. Eltaher, A.E. Alshorbagy, E.I. Meletis, Static analysis of nanobeams including surface effects by nonlocal finite element, J. Mech. Sci. Technol. 26 (2012) 3555-3563.

[25] F. Yang, Surface effects on large deflection of nanowires, J. Cent. South. Univ. T. 22 
(2015) 2060-2065.

[26] L. Wang, Vibration analysis of fluid-conveying nanotubes with consideration of surface effects, Phys. E 43 (2010) 437-439.

[27] G.F. Wang, X.Q. Feng, Effect of surface stresses on the vibration and buckling of piezoelectric nanowires, Europhys. Lett. 91 (2010) 56007.

[28] B. Gheshlaghi, S.M. Hasheminejad, Surface effects on nonlinear free vibration of nanobeams, Compos. Part B-Eng. 42(4) (2011) 934-937.

[29] R. Ansari, S. Sahmani, Bending behavior and buckling of nanobeams including surface stress effects corresponding to different beam theories, Int. J. Eng. Sci. 49 (2011) 12441255.

[30] R. Nazemnezhad, M. Salimi, S.H. Hashemi, P.A. Sharabiani, An analytical study on the nonlinear free vibration of nanoscale beams incorporating surface density effects, Compos. Part B-Eng 43 (2012) 2893-2897.

[31] P. Malekzadeh, M. Shojaee, Surface and nonlocal effects on the nonlinear free vibration of non-uniform nanobeams, Compos. Part B-Eng. 52 (2013) 84-92.

[32] S.H. Hashemi, R. Nazemnezhad, An analytical study on the nonlinear free vibration of functionally graded nanobeams incorporating surface effects, Compos. Part B-Eng. 52 (2013) 199-206.

[33] K.M. Hu, W.M. Zhang, Z.Y. Zhong, Z.K. Peng, G. Meng, Effect of surface layer thickness on buckling and vibration of nonlocal nanowires, Phys. Lett. A 378 (2014) 650-654.

[34] H. Jiang, C. Wang, Y. Luo, Vibration of piezoelectric nanobeams with an internal residual stress and a nonlinear strain, Phys. Lett. A 379 (2015) 2631-2636.

[35] G.F. Wang, X.Q. Feng, Timoshenko beam model for buckling and vibration of nanowires with surface effects, J. Phys. D Appl. Phys. 42 (2009) 155411.

[36] Z. Yan, L.Y. Jiang, The vibrational and buckling behaviors of piezoelectric nanobeams with surface effects, Nanotechnology 22 (2011) 245703.

[37] Y. Li, J. Song, B. Fang, J. Zhang, Surface effects on the postbuckling of nanowires, J. 
Phys. D Appl. Phys. 44 (2011) 425304.

[38] G.F. Wang, F. Yang, Postbuckling analysis of nanowires with surface effects, J. Appl. Phys. 109 (2011) 063535.

[39] H.S. Park, Surface stress effects on the critical buckling strains of silicon nanowires, Comp. Mater. Sci. 51 (2012) 396-401.

[40] C. Juntarasaid, T. Pulngern, S. Chucheepsakul, Bending and buckling of nanowires including the effects of surface stress and nonlocal elasticity, Phys. E 46 (2012) 68-76.

[41] Y. Li, C. Chen, B. Fang, J. Zhang, J. Song, Postbuckling of piezoelectric nanobeams with surface effects, Int. J. Appl. Mech. 4 (2012) 1250018. 


\section{Captions of the figures:}

Fig. 1. Schematic representation of magnetically actuated double nanowires carrying electric current.

Fig. 2. Fundamental frequency as a function of interwire distance for different levels of the electric current: (a) SS, (b) CC; $\left(\bar{B}_{0}=50, \lambda=15, \bar{f}_{0}=\bar{B}_{0} \bar{I}_{0}, \bar{f}_{1}=\left(\frac{\bar{I}_{0}}{\bar{d}}\right)^{2} ;(--\right.$

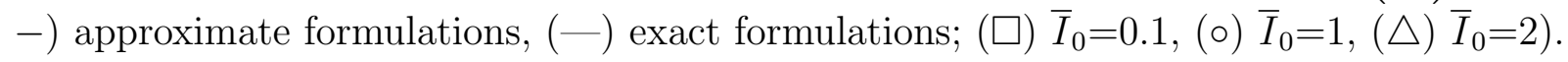

Fig. 3. Fundamental frequency as a function of slenderness ratio for different levels of the electric current: (a) SS, (b) CC; $\left(\lambda^{*}=30, \bar{B}_{0}^{*}=75, d=4 r_{0} ; \bar{I}_{0}=\bar{I}_{0}^{*}\left(\frac{\lambda}{\lambda^{*}}\right), \bar{B}_{0}=\bar{B}_{0}^{*}\left(\frac{\lambda}{\lambda^{*}}\right)^{2}\right.$; $(---)$ approximate formulations, (-) exact formulations; $(\square) \bar{I}_{0}^{*}=0.1$, (०) $\left.\bar{I}_{0}^{*}=1,(\triangle) \bar{I}_{0}^{*}=2\right)$.

Fig. 4. Fundamental frequency as a function of electric current for different levels of the slenderness ratio: (a) SS, (b) CC; $\left(\bar{B}_{0}^{*}=50, d=5 r_{0} ; \bar{I}_{0}=\bar{I}_{0}^{*}\left(\frac{\lambda}{\lambda^{*}}\right), \bar{B}_{0}=\bar{B}_{0}^{*}\left(\frac{\lambda}{\lambda^{*}}\right)^{2}\right.$; $(---)$ without surface effect, $(-)$ with surface effect; $(\square) \lambda^{*}=15$, (०) $\left.\lambda^{*}=20,(\triangle) \lambda^{*}=25\right)$.

Fig. 5. Fundamental frequency as a function of longitudinal magnetic field strength for different levels of the slenderness ratio: (a) SS, (b) CC; $\left(\bar{I}_{0}^{*}=0.2, d=5 r_{0} ; \bar{I}_{0}=\bar{I}_{0}^{*}\left(\frac{\lambda}{\lambda^{*}}\right)\right.$, $\bar{B}_{0}=\bar{B}_{0}^{*}\left(\frac{\lambda}{\lambda^{*}}\right)^{2} ;(---)$ without surface effect, $(-)$ with surface effect; $(\square) \lambda^{*}=15$, (о) $\left.\lambda^{*}=20,(\triangle) \lambda^{*}=25\right)$. 


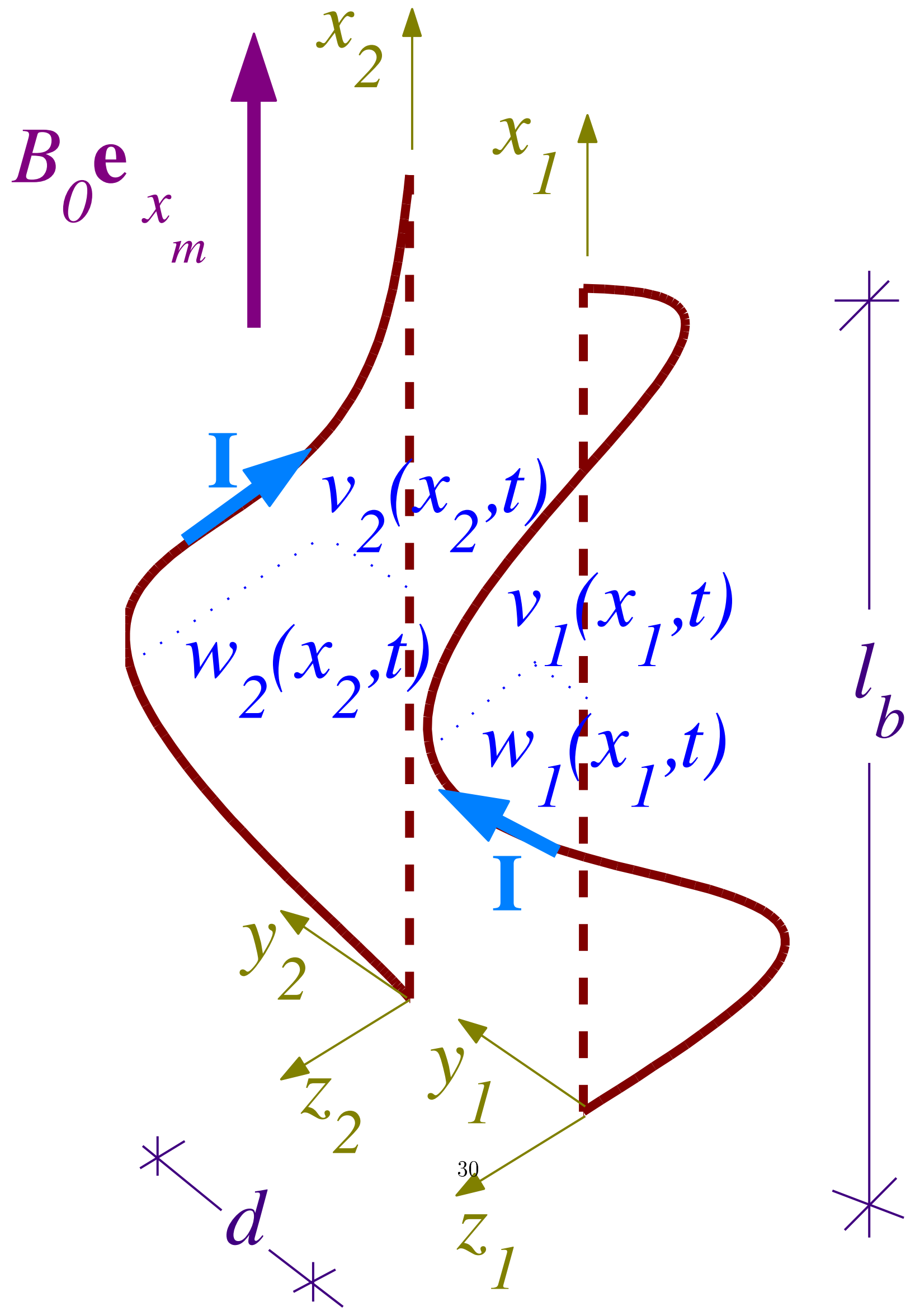

Fig. 1. 
(a)

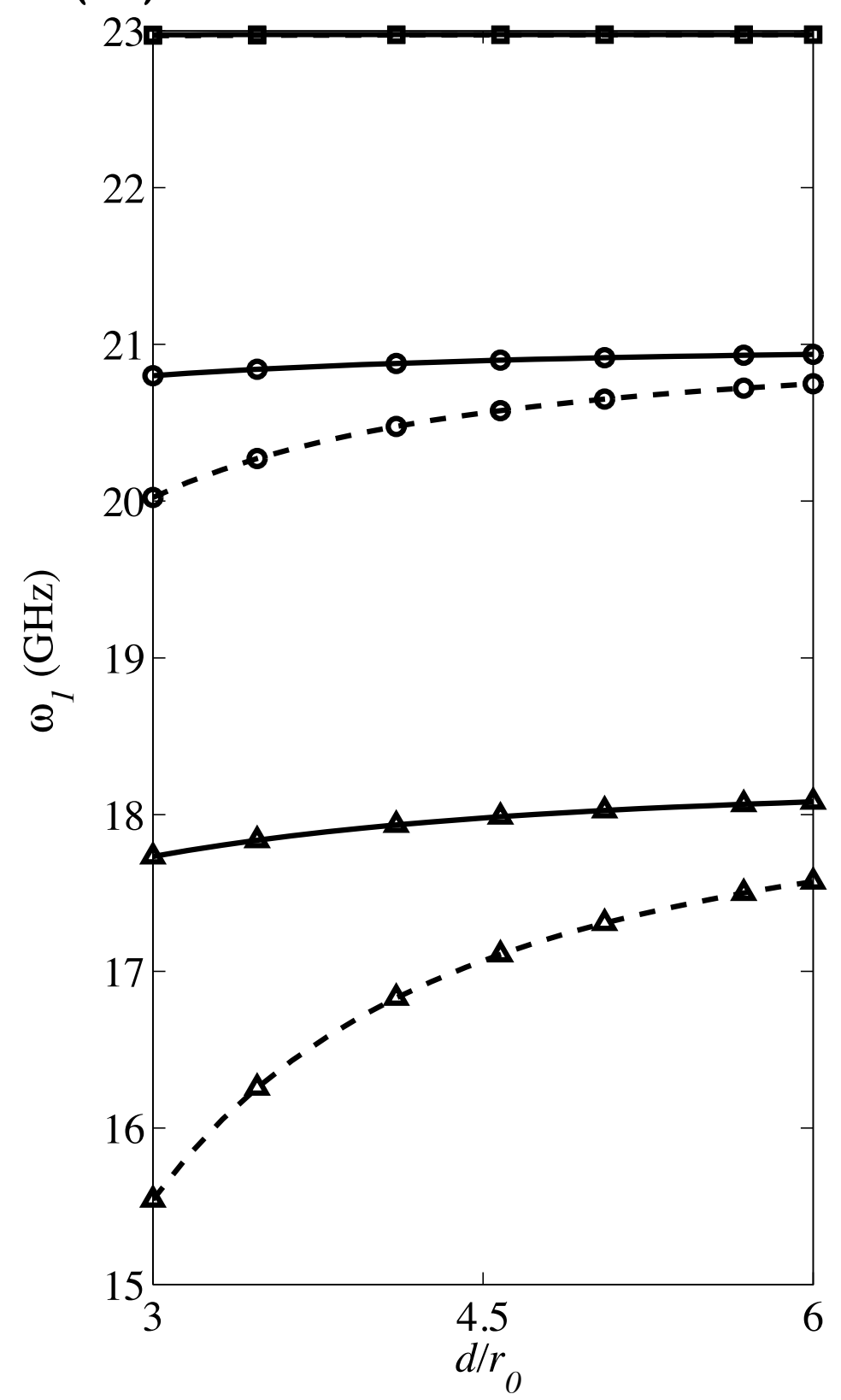

(b)

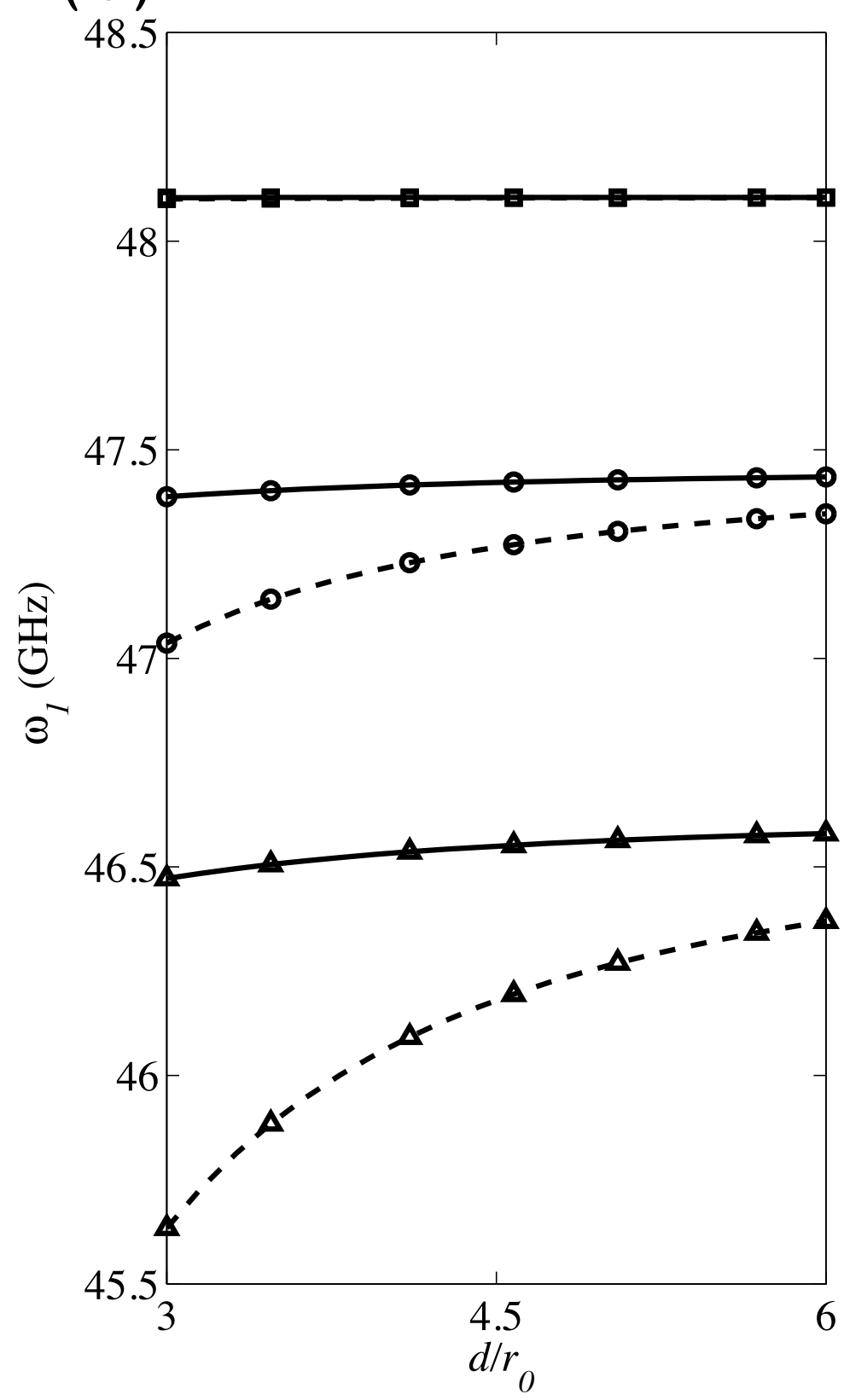

i 
(a)

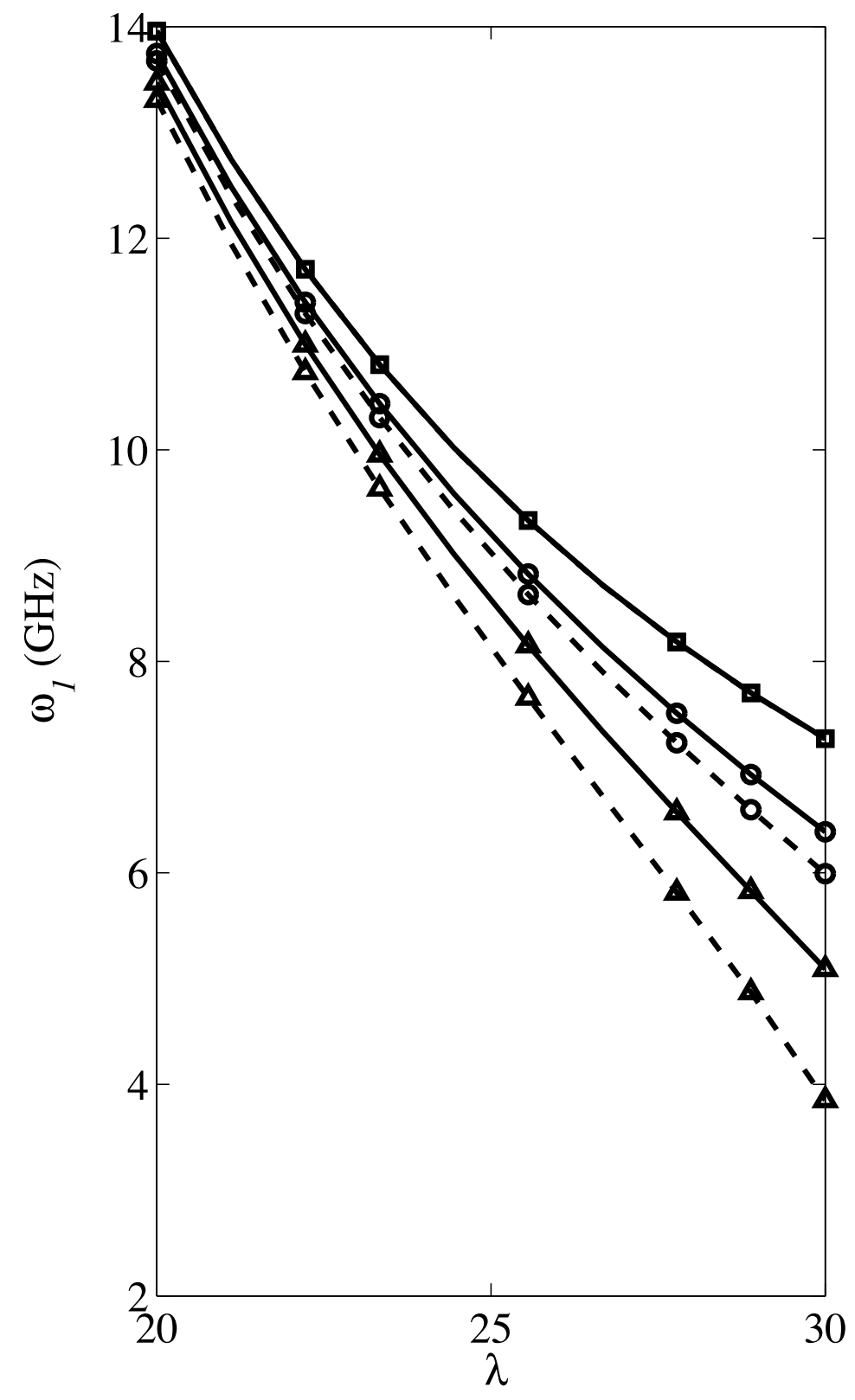

(b)

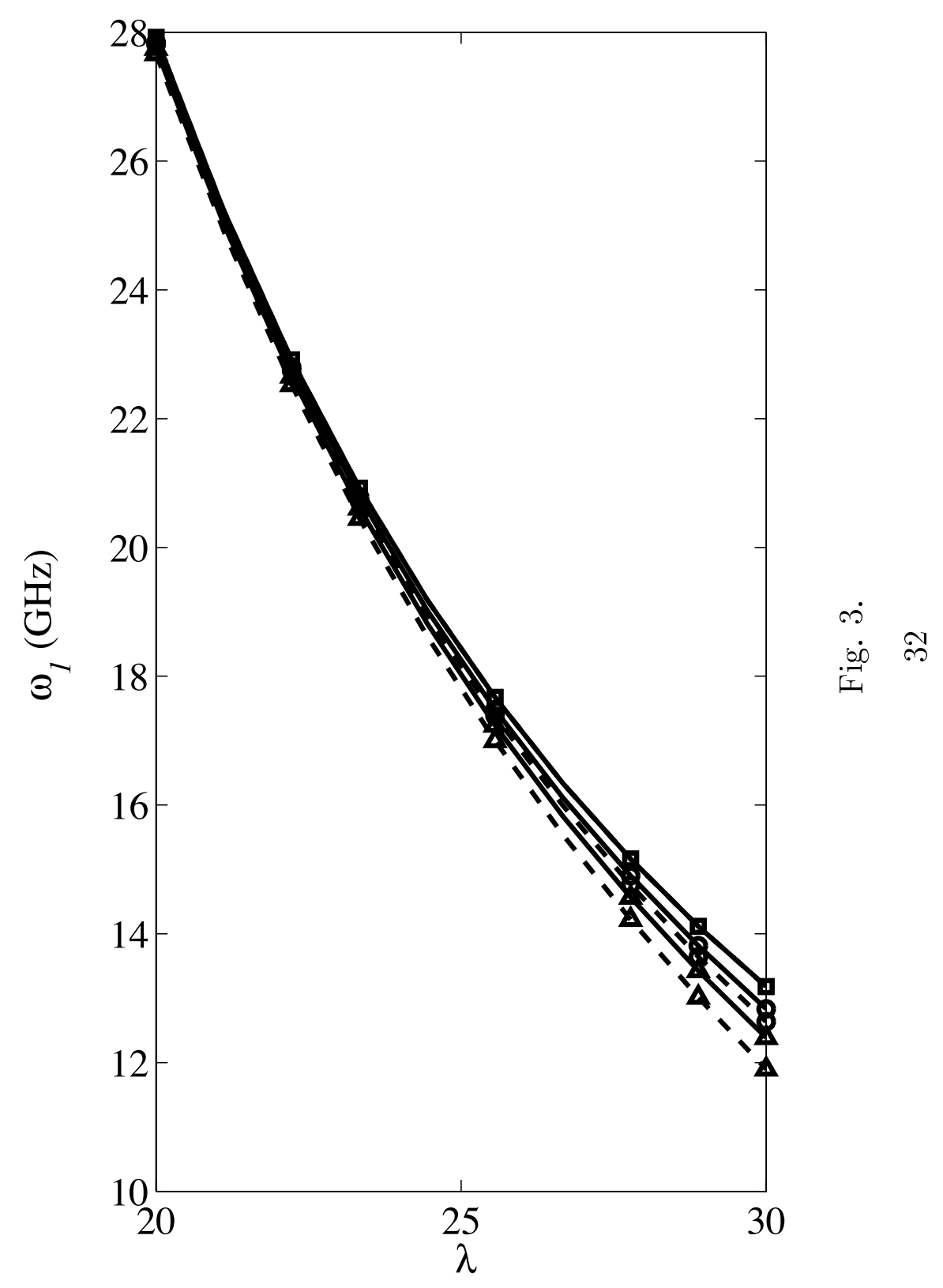


(a)

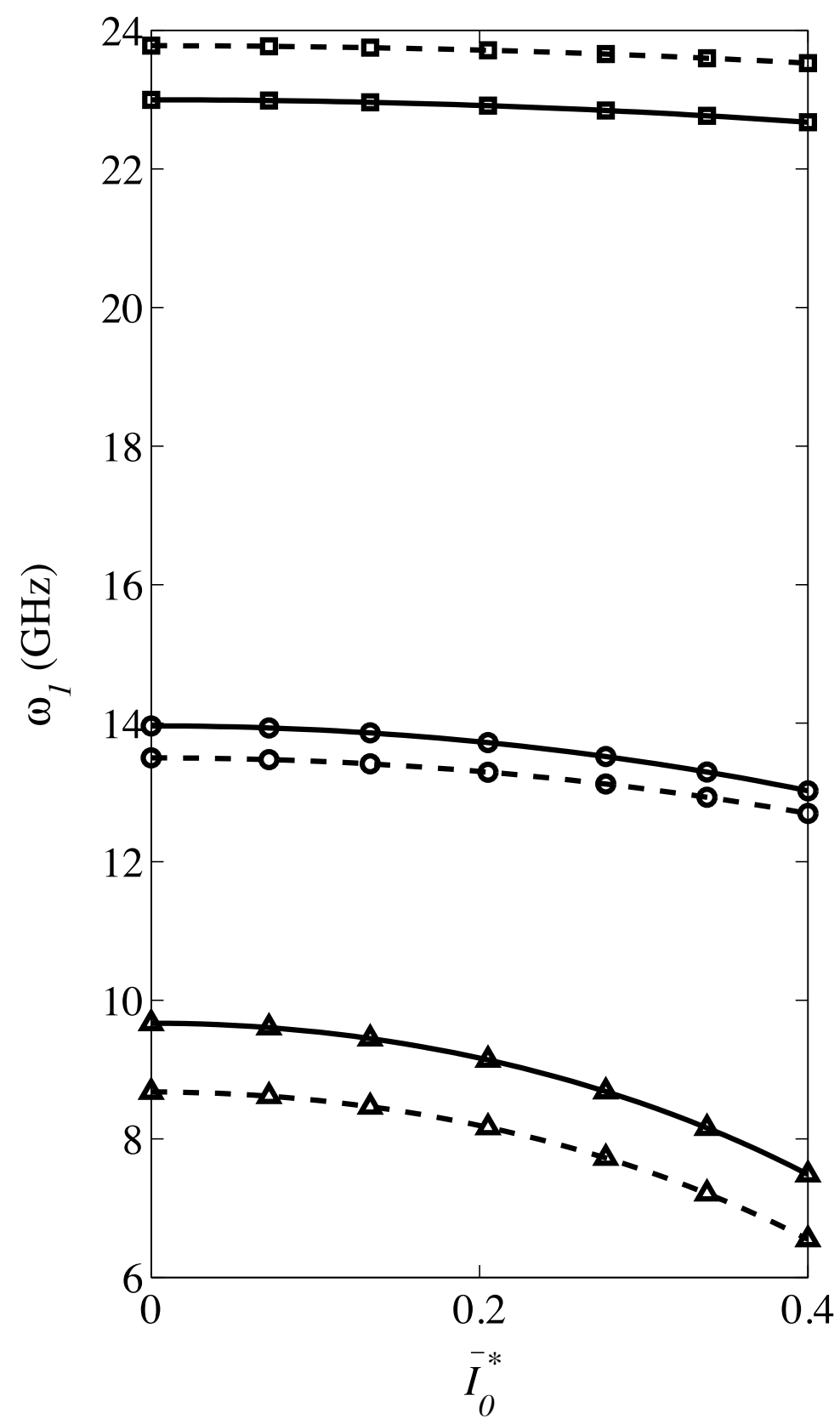

(b)

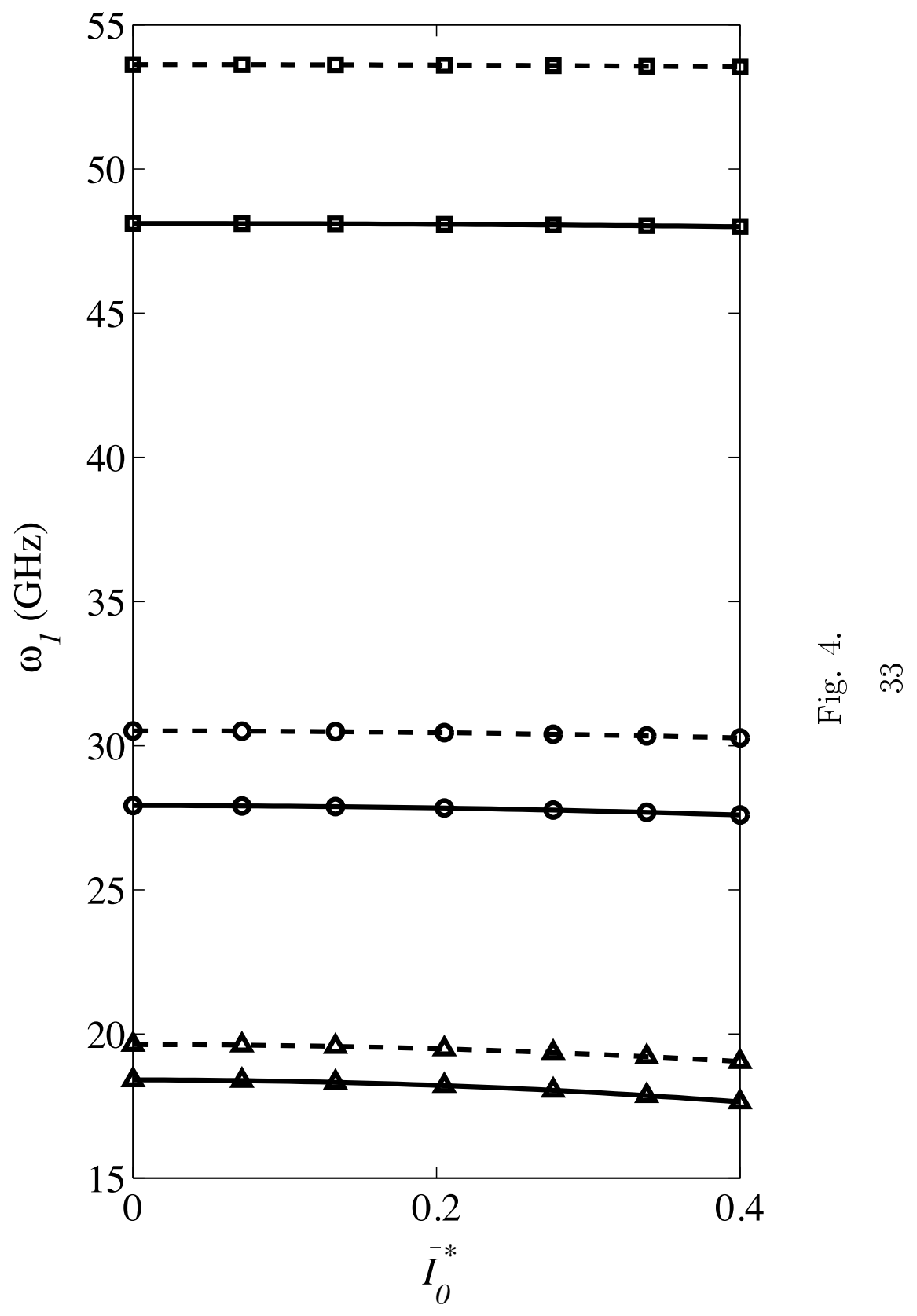


(a)

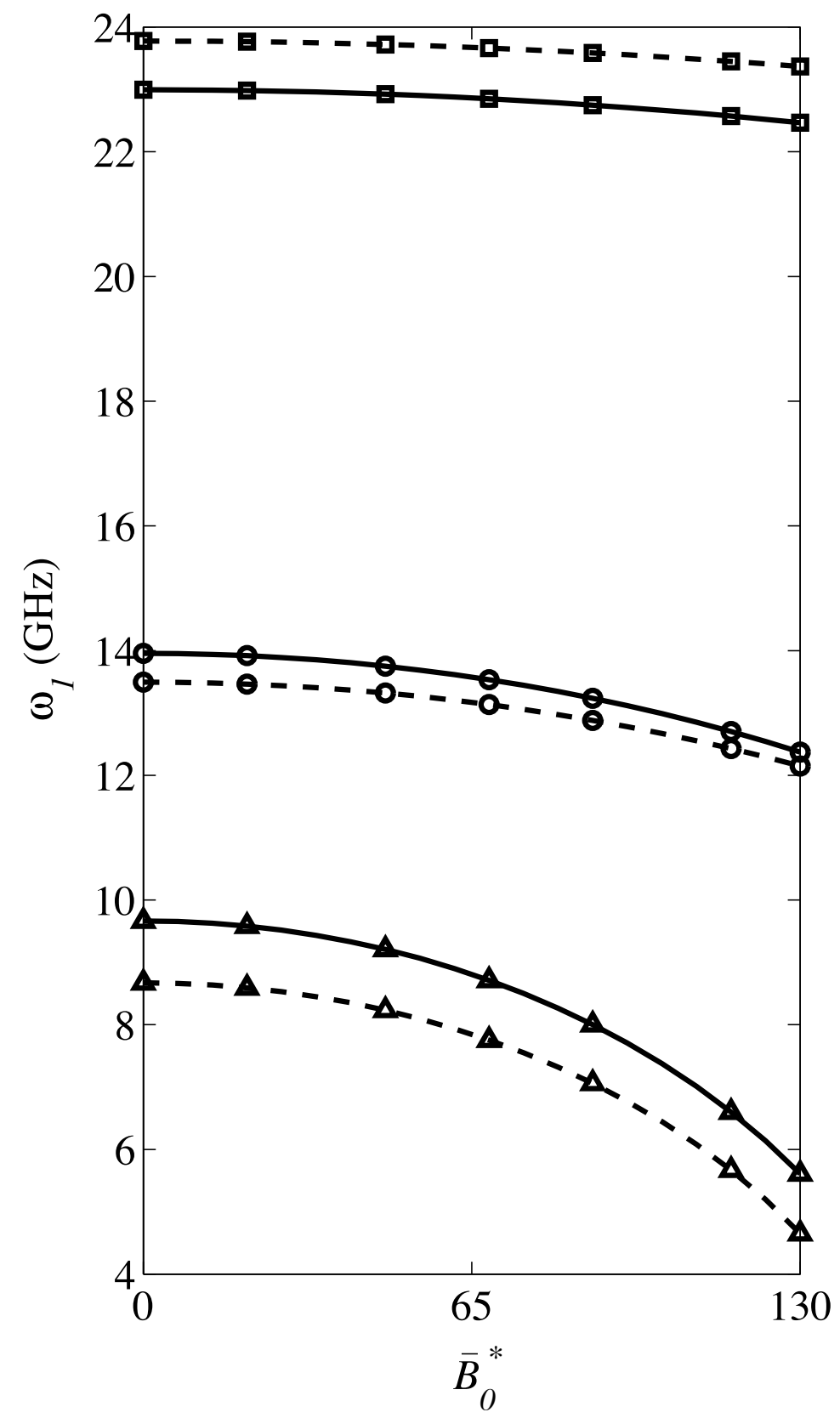

(b)

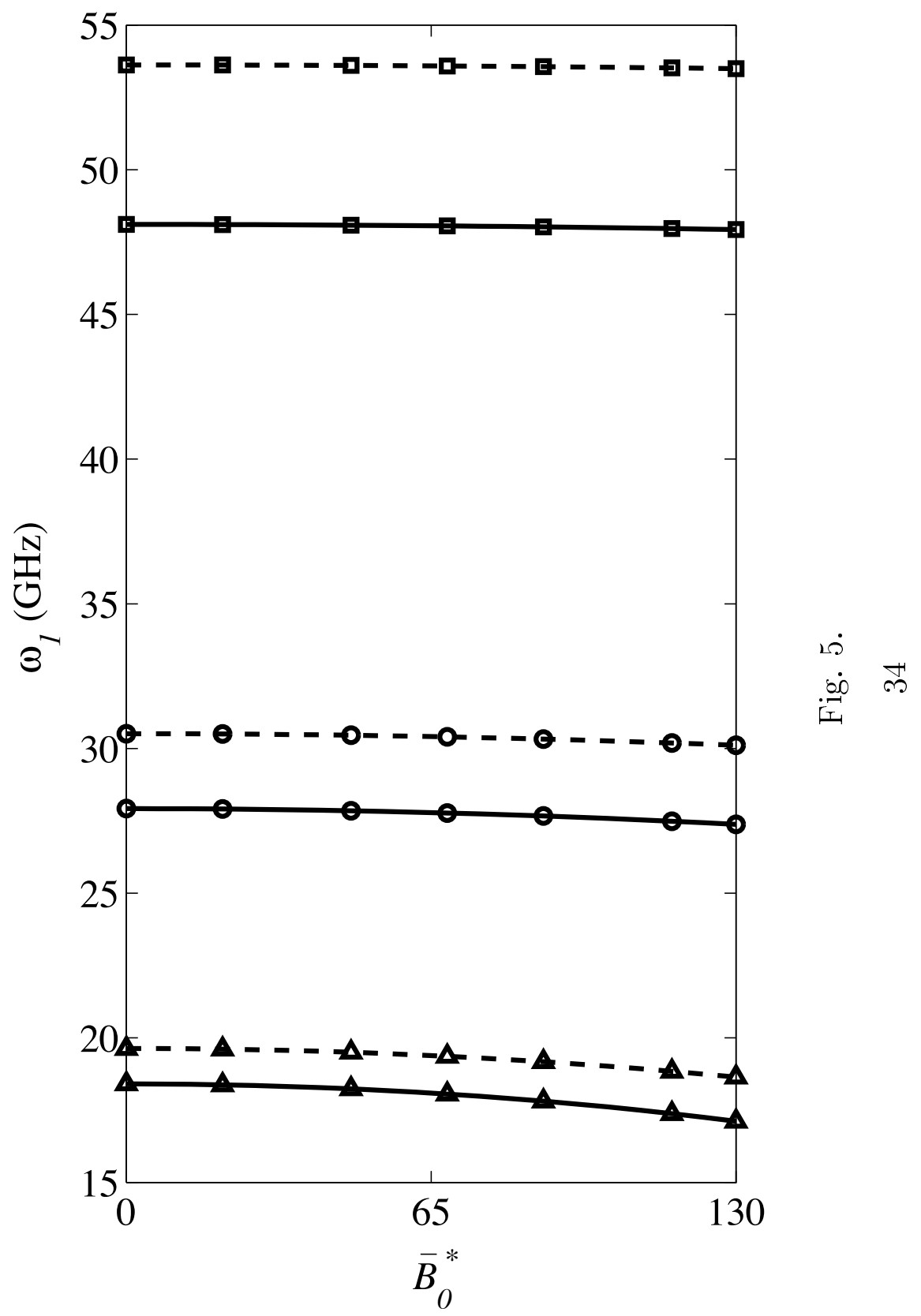

